\title{
Polizei und Automobilverkehr in Kaiserreich und Weimarer Republik
}

\author{
VON UWE FRAUNHOLZ
}

\begin{abstract}
Überblick
Als die ersten Autos zu Beginn des 20. Jahrhunderts erstmals in größerer Zahl auf den Straßen auftauchten, stießen sie auf erhebliche Gegenwehr. Insbesondere die Landbevölkerung reagierte mit Steinwürfen und Straßenblockaden. Hauptgründe für diese gewalttätige Opposition waren der sozial differenzierende Charakter des Autos sowie seine Auswirkungen auf die traditionellen Straßenbenutzer.

Dorfpolizisten und Landgendarmen übten eine rigorose Polizeiaufsicht über den Straßenverkehr aus, um die Konfliktpotentiale des frühen Automobilismus zu minimieren, und wurden auf diese Weise zu ,geborenen Feinden“ der Automobilisten, während sie gegenüber der unmotorisierten Bevölkerung eine eher schützende Haltung einnahmen. In diesem Zusammenhang lässt sich die regionale Varianz der Polizeidichte im Deutschen Kaiserreich auch durch die unterschiedliche Unfallgefahr der einzelnen Regionen erklären.

Mit zunehmendem Motorisierungsgrad in den 1920er Jahren veränderte sich die Haltung der Polizei in Richtung einer automobilfreundlicheren Einstellung, da die ökonomische Bedeutung des neuen Verkehrsmittels nun nicht mehr bestritten werden konnte. Dieser Prozess, der von der Ministerialbürokratie massiv forciert wurde, lässt sich durch eine Analyse von Werbeanzeigen aus der Automobilpresse nachzeichnen. Während die Inserate der Kaiserzeit Polizeibeamte vielfach karikierten und ihre Tätigkeit ironisierten, erkannte man nach dem Ersten Weltkrieg die Notwendigkeit der Verkehrsregulierung weitgehend an.
\end{abstract}

\footnotetext{
Abstract

When cars first appeared on the scene at the beginning of the $20^{\text {th }}$ century they were opposed by large segments of society. Especially rural residents reacted violently by throwing stones and blocking roads. Besides the disjunctive function of the motorcar one main reason for this violent opposition was its impact on other road users.

* Ich danke Jörg Baten, Alexander Moradi, Nikola Köpke sowie den Teilnehmern des 13. Polizeigeschichtlichen Kolloquiums (Enschede 2002) für wichtige Hinweise im Umfeld dieses Aufsatzes. Alle Irrtümer hat der Autor selbst zu vertreten.
} 
Local police authorities observed road traffic rigorously in order to minimize the conflicts of early automobilism. As a result subordinate policemen became „born enemies“ of automobilists with solicitous attitudes towards unmotorized inhabitants. Employing regression analysis it is shown that a high share of the variance of police density in the German Kaiserreich can be explained by the variable ,danger of accident" in the different regions.

With increasing of motorization in the 1920ies police authorities changed to automobile-friendly attitudes, since the economic importance of the new means of transportation could not be neglected anymore. This process was forced by ministry officials and is illustrated by an analysis of car-related advertisements: Whereas policemen were satirized in the Kaiserreich, their necessary function of traffic regulation was recognized by publicity in the Weimar Republic.

„Sagen wir es doch ganz offen: Der Schutzmann wird gehasst. Und selbst ganz einsichtige Männer verfallen gelegentlich ein wenig in den Blaukoller. Der Automobilist erst recht, der hält diesen Hass für ebenso erforderlich wie Benzin; der Schutzmann erscheint ihm als geschworener Feind, der ihn in die Autofalle locken will. Ist dieser Hass berechtigt? - Ja!“1

\section{Konfliktpotentiale des frühen Autoverkehrs}

Vor dem Ersten Weltkrieg fiel das Auto in Europa als Wirtschaftsfaktor kaum ins Gewicht. 1914 kam beispielsweise in Deutschland auf 698 Einwohner ein Kraftfahrzeug. Der Motorisierungsgrad erhöhte sich in der Weimarer Republik zwar dramatisch, so dass 193242 Einwohner auf jedes Kraftfahrzeug entfielen, die Massenmotorisierung wurde im Gegensatz zu den Vereinigten Staaten aber erst um 1970 erreicht. $^{2}$ Angesichts der vergleichsweise geringen Kaufkraft limitierten die hohen Preise und Unterhaltungskosten den Kreis potentieller Nachfrager in Deutschland stark, so dass sich die ersten Autofahrer vor allem aus adligen und großbürgerlichen Sportsleuten rekrutierten, die auch die repräsentativen und distinktiven Funktionen des neuen Verkehrsmittels schätzten. Aufgrund fehlender automobiler Infrastruktur wurde die Organisation in Automobilclubs, die teilweise die Benzinversorgung sicherstellten, sich ansonsten aber vor allem als schlagkräftige Interessenvertretung verstanden,

1 Automobil-Welt 1, 1903, S. 122.

2 Zur Frühgeschichte der Motorisierung vgl. Christoph M. Merki, Der holprige Siegeszug des Automobils 1895-1930. Zur Motorisierung des Straßenverkehrs in Frankreich, Deutschland und der Schweiz, Wien, Köln, Weimar 2002 sowie Barbara Haubner, Nervenkitzel und Freizeitvergnügen, Automobilismus in Deutschland 1886-1914, Göttingen 1998. Umfassend und mit aktuellen Bezügen: Kurt Möser, Geschichte des Autos, Frankfurt a.M. 2002. 
zur Notwendigkeit. Eine Analyse der Mitgliedschaften dieser Automobilvereine verdeutlicht die großbürgerliche Verankerung des frühen Automobilismus. Stets bildeten besitzbürgerliche Kreise einen Schwerpunkt der Mitgliedschaft, allenfalls in der Vorläuferorganisation des ADAC, der Deutschen MotorfahrerVereinigung (DMV), hatten mittelständische Mitglieder einiges Gewicht. Darüber hinaus war Autobesitz, wiederum im Gegensatz zu den Vereinigten Staaten, ein weitgehend städtisches Phänomen. ${ }^{3}$

Trotz dieser Exklusivität des frühen Automobilismus entfalteten die ersten Autos, als sie um die Wende zum 20. Jahrhundert erstmals in größerer Zahl im Straßenbild auftauchten und von den besitzenden Bürgern für längere Touren ins Umland genutzt werden konnten, ihre Verkehrswirksamkeit. Auf den ungeteerten Landstraßen riefen sie riesige Staubwolken hervor, die Passanten und Feldfrüchte beschmutzten und die wegeunterhaltungspflichtigen Gemeinden zu vermehrten Investitionen in Straßenbau und -unterhalt zwangen. Auch die hohe Unfallhäufigkeit des Autoverkehrs war beklagenswert. Die absoluten Zahlen der Verkehrsunfälle, Verletzten und Getöteten waren zwar aufgrund des geringen Motorisierungsgrades im Vergleich zu späteren Zeiten marginal; die noch nicht durch den täglichen Unfalltod abgestumpfte Bevölkerung und die Tagespresse reagierten trotzdem mit Empörung und dem Ruf nach verstärkter polizeilicher Überwachung. Analysen der Unfallursachen schienen die Kritiker des Autoverkehrs zu bestätigen. Etwa zwei Drittel aller Unfälle waren, entgegen den Beteuerungen der Autofahrer und Interessenverbände, auf Fahrfehler und überhöhte Geschwindigkeiten zurückzuführen und forderten vor allem Opfer unter den unmotorisierten Verkehrsteilnehmern, die keine „Knautschzone“ aufwiesen. Dabei konzentrierten sich die Autounfälle vor allem auf die Großstädte. In den ersten Jahren der polizeilichen Erfassung entfiel fast die Hälfte aller Autounfälle in Deutschland auf Berlin. Dort waren statistisch gesehen vor dem Ersten Weltkrieg bis zu 90 von 100 zugelassenen Kraftfahrzeugen jährlich in einen Unfall verwickelt.

Hinzu kam die Rücksichtslosigkeit vieler Herrenfahrer, die sich zu einem nicht unbeträchtlichen Maß nach Unfällen einer möglichen Bestrafung durch Flucht entzogen. Der Anteil von Fahrerfluchtsfällen halbierte sich zwischen 1906 und 1913 zwar nahezu, trotzdem kam es 1912/13 noch nach jedem fünfzehnten Unfall zur Fahrerflucht oder zum Versuch dazu. Der Anteil der nach Unfällen gegen Autofahrer verhängten Polizeistrafen und eröffneten Gerichtsverfahren blieb dagegen relativ konstant. Nach ungefähr einem Drittel aller Unfälle wurde gegen den Autofahrer behördlicherseits vorgegangen. Die Häufigkeiten von Polizeistrafen und Gerichtsverfahren standen dabei annä-

3 Zu den frühen Automobilclubs vgl. Barbara Haubner (wie Anm. 2), S. 67-105, zur sozialen Schichtung der Autofahrergemeinde Uwe Fraunholz, Motorphobia. Anti-automobiler Protest in Kaiserreich und Weimarer Republik, Göttingen 2002, S. 47-51. 
hernd im Verhältnis eins zu drei. ${ }^{4}$ Das Überwiegen der Gerichtsverfahren war vor allem darauf zurückzuführen, dass eine gerichtliche Klärung nötig wurde, wenn die Autofahrer gegen eine Polizeistrafe Einspruch erhoben.

Die Motorpresse erkannte das Problem der Fahrerflucht, bemühte sich jedoch, die hohe Rate den Unmotorisierten anzulasten: „Nicht weil der Kraftfahrer flüchtet, wird er verprügelt, sondern weil er nicht verprügelt werden will, flüchtet er.“ Stets nehme das Straßenpublikum nach Unfällen eine ,drohende Haltung" gegen die Autofahrer ein. ${ }^{5}$ Allerdings galt es, gerade um möglicher Lynchjustiz vorzubeugen, laut Kraftfahrzeuggesetz nicht als Fahrerflucht, wenn sich ein unfallverursachender Autofahrer erst einen Tag nach dem Vorfall bei der Polizei meldete und in einem Akt tätiger Reue Selbstanzeige erstattete. Auch eine drohende Beschädigung des wertvollen Fahrzeugs durch die „Volkswut“" galt zunächst als entschuldbarer Fluchtgrund. ${ }^{6}$

Das häufige Scheuen der Pferde vor den ungewohnten Gefährten sowie die bis dahin unbekannt hohen Geschwindigkeiten trugen ebenfalls dazu bei, dass sich aus Klassengegensätzen und antistädtischen Ressentiments gespeiste Konfliktpotentiale entwickelten, die sich vielerorts in Steinwürfen und gegen den Autoverkehr gerichteten Barrikadenbauten entluden. ${ }^{7}$ Bereits 1901 drohte der Berliner Polizeipräsident an, Straßen für den Kraftfahrzeugverkehr zu sperren, wenn die Autofahrer ihre Fahrgeschwindigkeit nicht minderten. Eine Besserung schien auszubleiben, so dass der preußische Innenminister 1907 vom Berliner Polizeipräsidenten empfindliche Strafen gegen schnellfahrende Autofahrer fordern musste. In einer öffentlichen Bekanntmachung wandte sich der Polizeipräsident daraufhin erneut direkt an die Autofahrer und forderte diese zur Reduzierung des Tempos auf. In der Folgezeit war zwar eine schärfere Verfolgung schnellfahrender Autofahrer zu beobachten, doch musste der Berliner Polizeipräsident in den frühen 20er Jahren seine Straßenaufsichtsbeamten erneut anweisen, gegen übermäßig schnelles Fahren „unnachsichtlich" einzuschreiten. ${ }^{8}$

4 Fraunholz (wie Anm. 3), S. 64, 73; Statistisches Jahrbuch d. Deutschen Reiches, 1914, S. 141; Bundesarchiv, R 1501 Nr. 13929, Blatt 126, Sammelstelle für Nachrichten über Führer von Kfz, Versagung und Entziehung von Führerscheinen 1910-1913.

5 Vgl. Automobil-Welt 21, 1923, Nr. 38, S. 24.

6 Vgl. Automobil-Welt 22, 1924, Nr. 9, S. 9; Motor, 1929, Nr. 7, S. 59f.; 1931, Nr. 5, S. 7f.; Heinz Emil Wendel, Auto und Kraftrad. Nach den Vorschriften für das Reichsgebiet, Berlin 1927, S. 47.

7 Vgl. Uwe Fraunholz (wie Anm. 3) sowie für die Schweiz Christoph M. Merki, Den Fortschritt bremsen? Der Widerstand gegen die Motorisierung des Straßenverkehrs in der Schweiz, in: Technikgeschichte 65, 1998, S. 233-253.

8 Landesarchiv Brandenburg, PrBrRep 30 Berlin C, tit. 133, Nr. 76, Bd 7 (18790), Minister des Inneren an Polizeipräsident Berlin, 22. 6. 1907; Landesarchiv Brandenburg, PrBrRep 30 Berlin C, tit. 133, Nr. 134, Bd 1, Blatt 9, Bekanntmachung des Berliner Polizeipräsidenten, 27. 6. 1908. 
Die Automobilpioniere trafen somit neben einer in weiten Teilen autokritischen Öffentlichkeit und der teilweise gewalttätig reagierenden Landbevölkerung auch auf Ordnungskräfte, die den verbreiteten Unmut über die Autos $\mathrm{zu}$ administrieren schienen. In so genannten „Autofallen“ versteckten sich Dorfpolizisten und Landgendarmen, um Autofahrer zu protokollieren, welche die rigiden Tempolimits überschritten. Dass dabei Kreise in Konflikt mit dem Gesetz kamen und zuweilen in einen „Blaukoller“ verfallen konnten, die sich polizeilicher Observanz ansonsten kaum ausgesetzt sahen, ermöglicht neue Einsichten in die Praxis staatlicher Überwachung. Für die Automobilisten blieb es jedenfalls unverständlich, dass den Aussagen subalterner Polizisten mehr Gewicht beigemessen wurde als den Darlegungen von Zeugen, die den Spitzen der Gesellschaft zuzurechnen waren. Auch der Verweis auf die nachgeordnete soziale Stellung der Gendarmen konnte das Vertrauen in den Beamteneid nicht erschüttern.

Die Hilflosigkeit der Autofahrer gegenüber scheinbarer polizeilicher Willkür und Allmacht wurde in ironisierende und karikierende, bildliche Darstellungen der Verkehrspolizei umgesetzt. Unterstellt man den Fachzeitschriften und ihren Rezipienten übereinstimmende Deutungen der Straßenrealität, so lässt sich jedoch durch die systematische Analyse von Werbeanzeigen aus der Automobilpresse die allmähliche Anerkennung der Nützlichkeit polizeilicher Verkehrsregulierung nachzeichnen (vgl. Abschnitt 4). Bei der Erweiterung der ikonographischen Analyse und ikonologischen Interpretation um sozialhistorische Dimensionen steht zwar die Entwicklung des Bildmotivs Verkehrspolizei im Vordergrund, die Aneignung durch den Bildbetrachter wird aber im Falle der in hohem Maße zielgruppenspezifischen Produktwerbung in Fachzeitschriften mitgedacht. Die Darstellung des Verhältnisses zwischen Polizist und Autofahrer in Inseraten der automobilen Zulieferindustrie dürfte demnach weitgehend dem Selbstbild der Herrenfahrer entsprechen. ${ }^{9}$

\section{Observanz über den Autoverkehr und Polizeidichte im Kaiserreich}

Klagen der Autofahrer über die Behandlung durch die Polizeikräfte und die Einrichtung von Stoppstrecken füllten die frühen Automobilzeitschriften, wobei den Beamten vielfach Pedanterie in der Auslegung von Verkehrsvorschriften unterstellt wurde. Tatsächlich war die Gefahr für Autofahrer vor dem Ersten Weltkrieg relativ groß, mit Strafmandaten belegt zu werden. 1912 wurden beispielsweise in München gegen 324 der 475 registrierten Chauffeure insgesamt 909 Strafverfügungen erlassen. Die Summe der Strafgelder be-

9 Zur Nutzbarmachung bildlicher Quellen in der Sozialgeschichte vgl. Heike Talkenberger, Von der Illustration zur Interpretation. Das Bild als historische Quelle. Methodische Überlegungen zu einer historischen Bildkunde, in: Zeitschrift für Historische Forschung 21, 1994, S. 289-313, S. 293-297. 
trug über 6700 Mark. ${ }^{10}$ Die Praxis polizeilicher Überwachung des Autoverkehrs in Deutschland führte gar zu internationalen Verwicklungen. 1906 berichtete der New York Herald über die ,schlagende Ungerechtigkeit der voreingenommenen Behörden“ und die ,aggressive feindliche Taktik“ des Fallenbetriebs in Baden, die sich zu einem „öffentlichen Skandal“" ausgeweitet hätten. Das Blatt rief zum Boykott Deutschlands auf, da das Einziehen von Geldstrafen unter Waffengewalt eines zivilisierten Volkes unwürdig sei, das Verhalten der Polizeikräfte auf „blinden Vorurteilen“ gegen den Autoverkehr und dem „Biereifer" der Beamten beruhe. ${ }^{11}$

Beschwerden über ähnliche Zustände gingen der Automobilpresse und den Clubs in großer Zahl aus allen Regionen des Deutschen Reichs zu. Insbesondere an den vielbefahrenen Strecken in der Umgebung der Großstädte Berlin, Hamburg, München, Frankfurt und Köln wurde gegen Geschwindigkeitsübertretungen scharf vorgegangen. Zahlreiche Anzeigen erfolgten auch wegen Rauchentwicklung, unterlassener Warnzeichen und ungenügender Beleuchtung. Die Autofahrer waren besonders darüber erbost, dass sich Polizisten versteckten, um Verkehrssünder aus dem „Hinterhalt“ zu protokollieren. Strafmandate gingen den Autofahrern zudem oft erst nach mehreren Wochen zu, so dass der Antritt eines Gegenbeweises erschwert wurde. ${ }^{12}$ Allerdings reagierten die Autofahrer oft nur mit einem Antrag auf gerichtliche Entscheidung, um den betreffenden Gemeinden Strafgelder zu entziehen, da im Fall einer gerichtlichen Verurteilung die Gelder der Staatskasse zuflossen. ${ }^{13}$

In den „Autofallen“ wurde in der Regel die Strecke zwischen zwei Bäumen abgeschritten und die benötigte Zeit der vorbeifahrenden Wagen mit der Taschenuhr gemessen. Die Ungenauigkeit dieser Methode erregte schärfsten Protest, zumal die örtlichen Behörden offenbar das relativ willkürliche Vorgehen ihrer Unterbeamten duldeten und die zusätzlichen Einnahmen für die Gemeindekasse gerne entgegennahmen. Die verhängten Strafen konnten dabei eine beträchtliche Höhe erreichen. Mit der Verabschiedung des Kraftfahrzeuggesetzes galten ab 1910 Höchststrafen bis zu 150 Mark oder sechs Wochen Haft. Die Höhe der Strafen traf vor allem angestellte Chauffeure, da sich Wagenbesitzer zuweilen weigerten, Strafmandate zu begleichen, und

10 Vgl. Das Auto-Recht 1, 1913, Nr. 9, S. 250f.

11 Vgl. Allgemeine Automobil Zeitung 7, 1906, Nr. 38, S. 66f.; Nr. 43, S. 62f.; 8, 1907, Nr. 8, S. 59ff.; Nr. 9, S. 62.

12 GStA PK I. HA, Rep. 77, tit. 1328, Nr. 32, Kaiserlicher Automobil-Club an preußischen Innenminister, 14. 11. 1908; Der Motorfahrer, 1909, S. 781. Zu den Autofallen vgl. auch Dietmar Fack, Automobil, Verkehr und Erziehung. Motorisierung und Sozialisation zwischen Beschleunigung und Anpassung 1885-1945, Opladen 2000, S. $178 \mathrm{f}$.

13 Die Aufteilung der Strafgelder unter Gemeinden, Landkreisen und der Staatskasse war uneinheitlich geregelt. In Bayern gingen Strafgelder auch ohne Gerichtsverhandlung an die Staatskasse. In Bremen wurde der Gemeinde das Strafgeld auch dann entzogen, wenn der Einspruch vor der mündlichen Verhandlung zurückgezogen wurde. Vgl. Allgemeine Automobil Zeitung 10, 1909, Nr. 34, S. 46f. 
sich die Strafen recht undifferenziert gegen Fahrer oder Autobesitzer richteten. Extreme Autogegner forderten bei Geschwindigkeitsübertretungen allerdings ,grundsätzlich Arreststrafen mit Vermögenseinbußen“. ${ }^{14}$

Außerdem wurde mit dem Kraftfahrzeuggesetz die Möglichkeit des Führerscheinentzugs eingeführt. Dabei war ein Entzug selbst bei außerhalb des Straßenverkehrs liegenden Delikten wie Eigentumsvergehen, fahrlässiger Körperverletzung, Tötungen, Rohheits- und Sittlichkeitsverbrechen möglich. Auch wenn man durch Alkoholabhängigkeit oder die „Neigung zu Ausschreitungen" auffällig wurde, konnte man den Führerschein verlieren. Allerdings machten die Behörden von der Möglichkeit des Führerscheinentzugs zunächst nur zurückhaltend Gebrauch. 1910 wurden lediglich 117 Führerscheine entzogen. Die Zahl der Entziehungen ging dann sogar leicht zurück, um 1913 auf 323 Fälle anzusteigen. Dabei wurde nur etwa ein Viertel der Führerscheine aufgrund der Übertretung polizeilicher Verkehrsvorschriften entzogen. ${ }^{15}$

Die Bemessung der zulässigen Höchstgeschwindigkeit orientierte sich zunächst an der Geschwindigkeit von Pferden und Fuhrwerken. Die Landbevölkerung hatte sich an die Beschleunigung des Straßenverkehrs noch keinesfalls gewöhnt und betrachtete die Automobile als Gefahr, auch wenn sie nach heutigen Maßstäben recht langsam fuhren. Während beispielsweise in Chemnitz die zulässige Geschwindigkeit eines Autos 1899, ,diejenige eines mässig trabenden Pferdes“ war, wurde in Mannheim das Tempo bereits modern definiert und innerorts auf $6 \mathrm{~km} / \mathrm{h}$ festgesetzt. Oft wurde aber willkürlich Schrittgeschwindigkeit vorgeschrieben. Die vielerorts unangemessen geringe Höhe der zulässigen Geschwindigkeit führte zu einer wahren Anzeigenflut, die zuweilen eine Vernachlässigung der übrigen Aufgaben der Polizeikräfte befürchten ließ. ${ }^{16}$

Nachdem 1908 bei einer Besprechung im Reichsamt des Innern 15 von 35 Firmen der Automobilindustrie die strengen polizeilichen Vorschriften und die Höhe der Strafmandate als Ursachen für die Krise ihrer Branche benannt hatten, reagierte man umgehend. Im Juli 1909 erklärten die zuständigen preußischen Ministerien in einem Erlass an die nachgeordneten Behörden, dass das versteckte Protokollieren von Autos den Amtspflichten preußischer Polizisten widerspräche. Übertretungen müssten durch präventive Warnungen vermieden

14 Vgl. Emil Jung, Radfahrseuche und Automobilenunfug. Ein Beitrag zum Recht auf Ruhe, München 1902, S. 39; Der Motorwagen, 1908, S. 840; Allgemeine Automobil Zeitung 8, 1907, Nr. 42, S. $58 f$.

151925 verlor ein Berufskraftfahrer beispielsweise den Führerschein, weil er in einer Strafsache wegen Jagdvergehens einen Zeugen zum Meineid verleitet hatte. Vgl. Der deutsche Kraftfahrer 3, 1927, Nr. 5, S. 14.

16 Vgl. GStA PK I. HA, Rep. 77, tit. 1712, Nr. 16a, Regierungspräsident Coblenz an Minister des Innern, 14. 4. 1913; Automobile, 1899, H. 6, S. 76; Bundesarchiv, R 3001, Nr. 7073, B1. 85, Kölner AC an Bundesrat, 14. 11. 1909. 
werden. Die Exekutivbeamten sollten daher Autofahrer im Übertretungsfall durch geeignete Signale warnen und zum Anhalten auffordern. ${ }^{17}$

Obwohl es in der Folgezeit nach vermeintlichen Missbräuchen mehrfach zum Entzug der Ortspolizeibefugnis kam, änderte sich bis zum Ersten Weltkrieg wenig an der alltäglichen Überwachungspraxis, und das versteckte Protokollieren konnte nie gänzlich unterbunden werden. Die Deutsche AutoLiga machte es sich daher zur Aufgabe, die polizeiliche Überwachung des Autoverkehrs ihrerseits zu überwachen. Mitarbeiter der Organisation warnten verschiedentlich nahende Autofahrer vor Stoppstrecken durch das Schwenken weißer Flaggen. Dieses Vorgehen wurde von den Behörden jedoch nicht immer akzeptiert. 1913 wurden Mitglieder des Norddeutschen AC, die vor einer Autofalle in Oldeslohe mit beschrifteten Schildern gewarnt hatten, wegen Preßvergehens und Sonntagsentheiligung verurteilt. ${ }^{18}$ Ein geeigneteres Mittel, die Polizeibehörden für die Bedürfnisse des Autoverkehrs zu sensibilisieren, fanden die Automobilclubs mit der Veranstaltung von Kontrollfahrten, an denen Polizeibeamte in Zivil teilnahmen. Damit wollte man auf das Ausmaß der Verkehrsverstöße unmotorisierter Verkehrsteilnehmer hinweisen, die Forderung nach konsequenterer Ahndung untermauern und Verständnis für den Kraftverkehr wecken. Gleichzeitig konnten Ausschreitungen und Übertretungen geahndet werden, ohne dass die Autofahrer Anzeigen erstatten mussten, was im Fall des einfachen Verstoßes gegen Polizeivorschriften ihrem Selbstverständnis als „Gentlemen“ widersprechen konnte. ${ }^{19}$

Die sich langsam durchsetzende Regelung, jeweils zwei abstoppende Polizisten für den „Fallendienst“ bereitzustellen, konfrontierte die Dienststellen mit erheblicher Personalnot. Allerdings scheint vor allem die durch den Autoverkehr bedingte Erhöhung der Unfallgefahren eine verstärkte Polizeipräsenz bewirkt zu haben. So lässt sich die unterschiedliche Polizeidichte in Deutschland - in Berlin entfielen 1907 etwa 29 Polizeiexekutivbeamte auf 10.000 Einwohner, während es in Anhalt nur knapp neun waren (s. Tabelle 1, S. 112/113) - auch durch die unterschiedliche Unfallgefährlichkeit der be-

17 Vgl. Bundesarchiv, R 1501, Nr. 13927a, Protokoll der Besprechung über Produktionserhebungen in der Automobilindustrie, 12. 9. 1908; Allgemeine Automobil Zeitung 11, 1910, Nr. 29, S. $46 f$.

18 Vgl. Allgemeine Automobil Zeitung 10, 1909, Nr. 37, S. 43; 14, 1913, Nr. 8, S. 10. Noch 1925 wurde ein Autofahrer wegen Beamtenbeleidigung angeklagt, weil er versucht hatte, einen preußischen Gendarmen in einer Autofalle zu fotografieren. Vgl. Allgemeine Automobil Zeitung 28, 1927, Nr. 11, S. 21f.

19 Über eine derartige Kontrollfahrt in Wiesbaden wurde 1907 berichtet: „Auf Veranlassung des Wiesbadener Automobilclubs haben in den letzten Tagen eine Anzahl von Kraftwagen in durchaus statthaftem Fahrtempo die Stadt die Kreuz und die quer durchfahren, um festzustellen, daß die Voreingenommenheit breiter Volksschichten wider diese Fahrzeuge eine hochgradige ist, und daß auch, ohne daß direkt irgend eine Veranlassung dazu gegeben wird, Ausschreitungen gegen die Automobile resp. deren Führer verübt werden“" (Der Kraftwagen, 1907, Nr. 19, S. 202f.). 


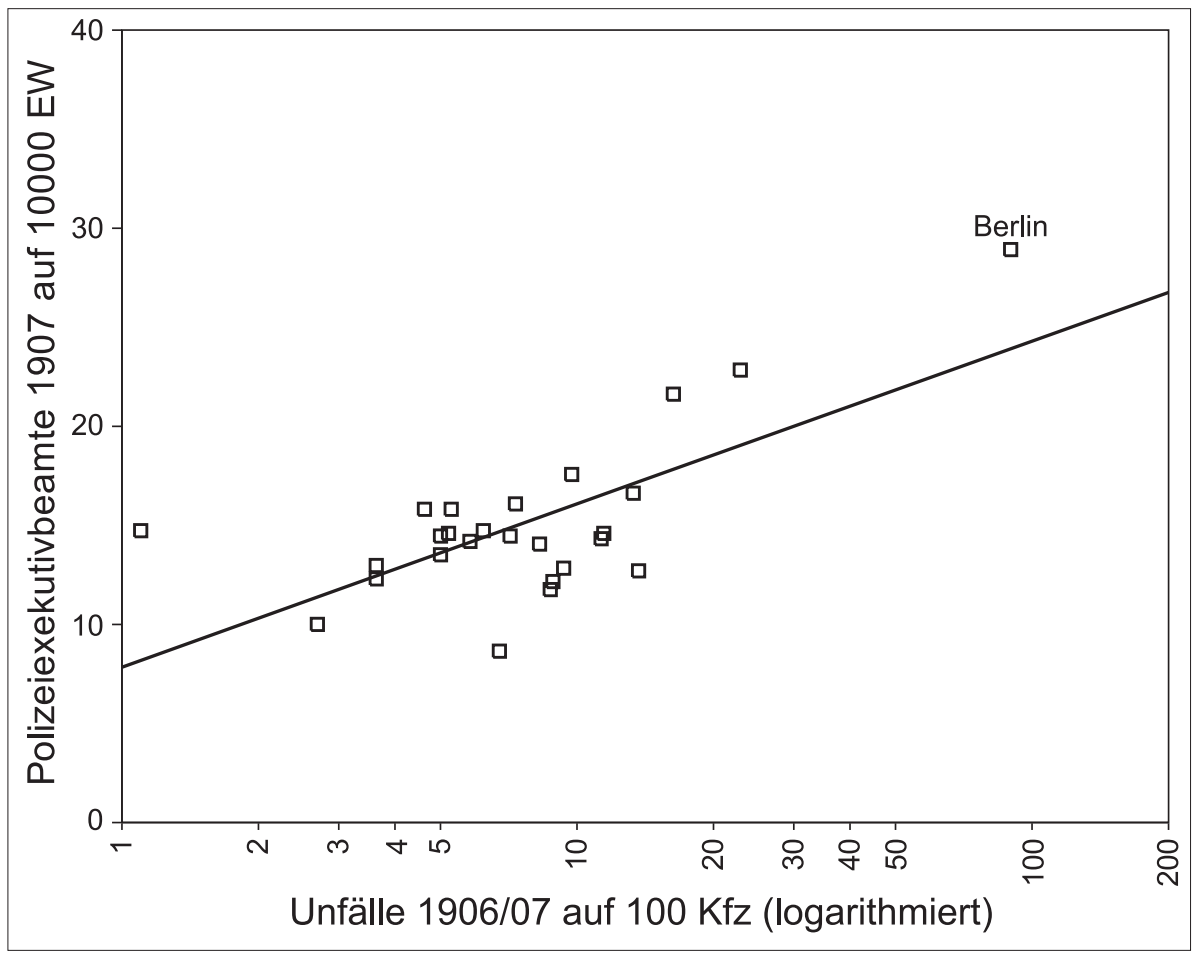

Schaubild 1: Streudiagramm zu Polizeidichte und Unfallgefahr

treffenden Regionen erklären. Im Streudiagramm (Schaubild 1) zeigt sich ein linearer Zusammenhang zwischen der Unfallgefährlichkeit und der Polizeidichte einer Region. Testet man verschiedene Erklärungen für die unterschiedliche Polizeidichte in Deutschland mittels Regressionsanalysen, setzt sich die Unfallgefährlichkeit - sowohl mit dem Ordinary Least Square-Verfahren als auch bei Gewichtung durch die Bevölkerung der Provinzen und Bundesstaaten - gegen die ansonsten für eine Erklärung herangezogene Variable des Anteils der großstädtischen Bevölkerung durch. Auch wenn bei der Interpretation aufgrund der geringen Fallzahlen Vorsicht geboten ist, dürfte sich hierin tatsächlich mehr abbilden als die erhöhte Unfallziffer der Großstädte. Da bis zu 47 Prozent der Varianz der Polizeidichte allein durch Unterschiede in den Unfallziffern des Autoverkehrs zu erklären sind, müssen herkömmliche Interpretationen, die allein obrigkeitsstaatliche Traditionen in der Polizierung verdächtiger Bevölkerungen als ursächlich für verstärkte Polizeipräsenz betonen, hinterfragt werden. ${ }^{20}$ Während der Anteil der großstädtischen Bevölke-

20 Eine Interpretation, die vor allem eine „Konzentration der Polizei in den Krisengebieten des Kaiserreichs“ betont, findet sich bei Wolfgang Knöbl, Polizei und Herrschaft im Modernisierungsprozeß. Staatsbildung und innere Sicherheit in Preußen, England und Amerika 1700-1914, Frankfurt a.M. 1998, S. 307; Ralph Jessen, Preußi- [Forts. auf S. 114] 


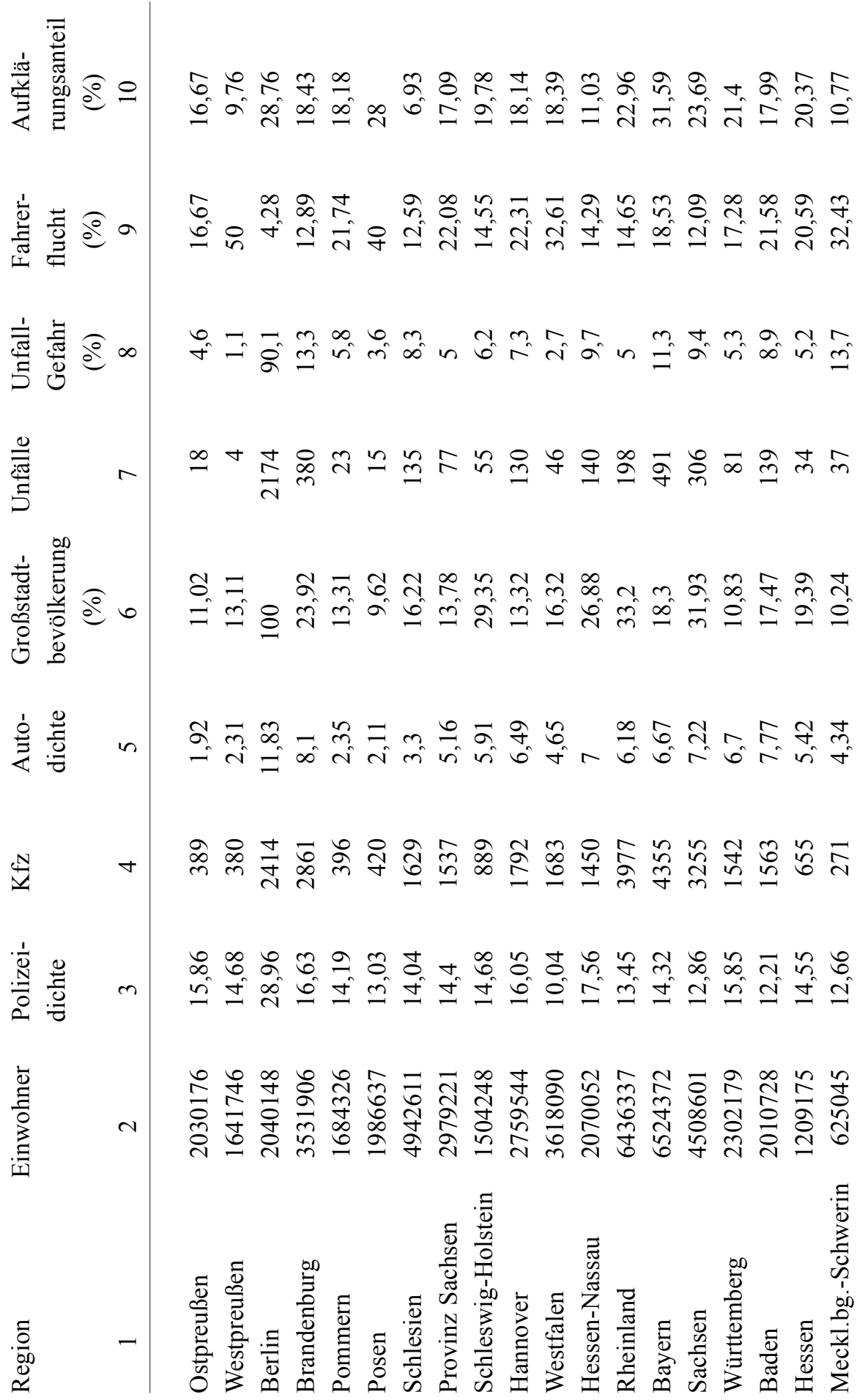




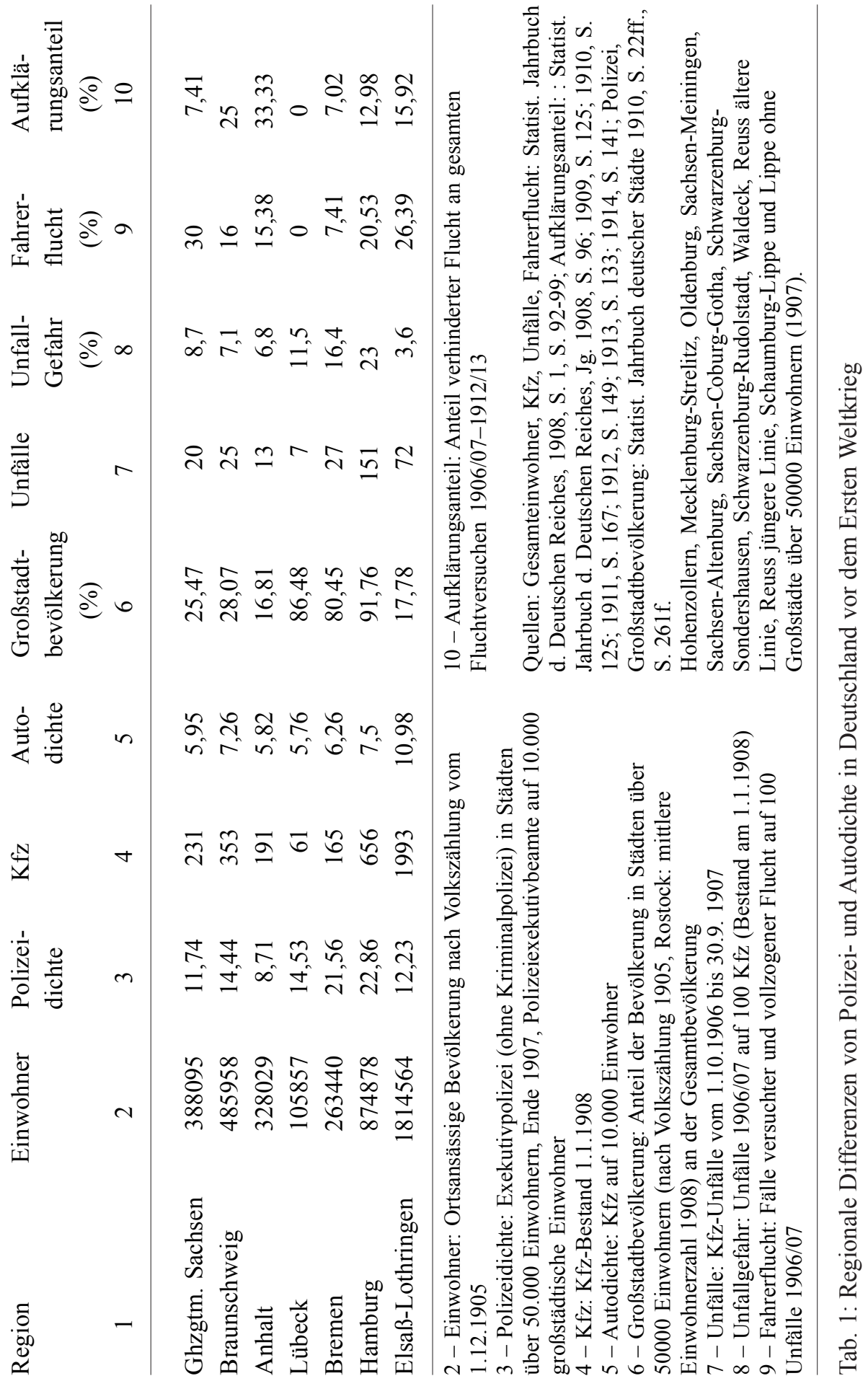


rung als Erklärung für die Varianz der Polizeipräsenz in einem der hier getesteten Modelle ebenfalls schwach signifikant wird, bleiben Autodichte und Rücksichtslosigkeit der Autofahrer - ausgedrückt in den auf 100 Autounfälle entfallenden Fahrerfluchtsfällen - als erklärende Variablen allerdings stets insignifikant. $^{21}$

Dürften also die zahlreichen Autounfälle die Polizeidichte erhöht haben, so lässt sich umgekehrt kaum behaupten, dass die verstärkte Präsenz der Ordnungshüter auf den Straßen alle Zumutungen des Autoverkehrs gemildert hat. Trägt man die Polizeidichte und die Aufklärungsrate bei der Fahrerflucht zwischen 1906 und 1913 (Anteil gescheiterter Fluchtversuche an Gesamtzahl der Fahrerfluchtsfälle) regional differenziert in einem Streudiagramm ab, so zeigt sich zunächst kein linearer Zusammenhang. Erst bei Unterscheidung Lübecks, Bremens, Hamburgs und Berlins von den eher ländlich geprägten Flächenstaaten, ergibt sich für die Großstädte ein positiver Zusammenhang zwischen der Polizeidichte und der Verhinderung von Fahrerflucht, der allerdings auf nur vier Beobachtungen beruht: Berlin wies bei höchster Polizeidichte den geringsten Anteil geglückter Fahrerflucht auf. Da dieser Befund aber dem negativen Zusammenhang zwischen Polizeidichte und verhinderter Fahrerflucht in den ländlichen Gebieten widerspricht, ist vor allem auf die Mitwirkung des Straßenpublikums bei der Festsetzung unfallverursachender Autofahrer in Großstädten hinzuweisen.

\section{Motorengeräusch als Sinfonie der Zeit: Weimarer Verkehrspolizei}

Im Ersten Weltkrieg schränkte man den privaten Kraftverkehr durch verschiedene Verordnungen schrittweise ein. Gleichzeitig wurde die zunehmende Anerkennung des kriegswichtigen Autos nun aber in Polizeiverordnungen deutlich, die das Verhalten der anderen Verkehrsteilnehmer reglementierten. Kutschern wurde ausdrücklich untersagt, Autos zu behindern und laut zu schimpfen. Zügel mussten straff gehalten werden. Überflüssiges Schwingen, Schlagen und Knallen mit der Peitsche war verboten. Die Fußgänger hatten sich auf dem Bürgersteig rechts zu halten und mussten rechts auswei-

sche Polizei und Arbeiterschaft im Kaiserreich, in: Peter Nischke (Hg.), Die Deutsche Polizei und ihre Geschichte. Beiträge zu einem distanzierten Verhältnis, Hilden 1996, S. 46-71 erkennt dagegen die Ambivalenz im Verhältnis zwischen Polizei und Arbeiterschaft: „Wenn man die Geschichte der preußischen Polizei mehr aus dieser sozialgeschichtlichen Perspektive sieht, verliert sie etwas ihre ,preußischen' Züge und man erkennt Aspekte institutioneller Modernisierung und Funktionsverlagerung" (S. 69).

21 In den hier vorgestellten Schätzmodellen wird nur die Exekutivpolizei (ohne Kriminalpolizei) in Städten über 50000 Einwohnern berücksichtigt. Verlässliche, regional differenzierende Angaben über die Stärke der Landgendarmerie wären für ein wirklichkeitsnäheres Bild einzubeziehen. Zur zeitlichen Entwicklung der Polizeidichte in Preußen bis 1913 vgl. Albrecht Funk, Polizei und Rechtsstaat. Die Entwicklung des staatlichen Gewaltmonopols in Preußen 1848-1914, Frankfurt a. M., New York 1986, S. 211-216. 


\begin{tabular}{|c|c|c|c|c|c|c|c|c|}
\hline & OLS 1 & WLS 1 & OLS 2 & WLS 2 & OLS 3 & WLS 3 & OLS 4 & WLS 4 \\
\hline 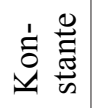 & $\begin{array}{l}2,34 * * * \\
(4,42)\end{array}$ & $\begin{array}{c}2,33 * * * \\
(4,17)\end{array}$ & $\begin{array}{l}2,16 * * * \\
(11,83)\end{array}$ & $\begin{array}{r}2,24 * * * \\
(13,04)\end{array}$ & $\begin{array}{c}2,08 * * * \\
(11,92)\end{array}$ & $\begin{array}{l}2,22 * * * \\
(12,81)\end{array}$ & $\begin{array}{r}2,30 * * * \\
(21,97)\end{array}$ & $\begin{array}{r}2,30 * * * \\
(28,00)\end{array}$ \\
\hline 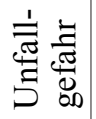 & $\begin{array}{l}0,11 \\
(1,50)\end{array}$ & $\begin{array}{r}0,18 * * \\
(2,53)\end{array}$ & $\begin{array}{c}0,15 * * \\
(2,30)\end{array}$ & $\begin{array}{c}0,19 * * * \\
(3,60)\end{array}$ & $\begin{array}{c}0,13^{*} \\
(1,97)\end{array}$ & $\begin{array}{c}0,17 * * * \\
(3,34)\end{array}$ & $\begin{array}{c}0,19 * * * \\
(3,96)\end{array}$ & $\begin{array}{c}0,19 * * * \\
(4,80)\end{array}$ \\
\hline 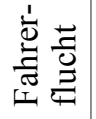 & $\begin{array}{l}-0,06 \\
(-0,56)\end{array}$ & $\begin{array}{l}-0,02 \\
(-0,18)\end{array}$ & - & - & - & - & - & - \\
\hline 定旁: & $\begin{array}{l}-0,15 \\
(-1,58)\end{array}$ & $\begin{array}{l}-0,10 \\
(-1,20)\end{array}$ & $\begin{array}{l}-0,13 \\
(-1,28)\end{array}$ & $\begin{array}{l}-0,11 \\
(-1,28)\end{array}$ & - & - & - & - \\
\hline 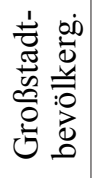 & $\begin{array}{c}0,183 * \\
(2,08)\end{array}$ & $\begin{array}{l}0,07 \\
(0,82)\end{array}$ & $\begin{array}{r}0,137 \\
(1,82)\end{array}$ & $\begin{array}{l}0,08 \\
(1,02)\end{array}$ & $\begin{array}{l}0,11 \\
(1,51)\end{array}$ & $\begin{array}{l}0,04 \\
(0,58)\end{array}$ & - & - \\
\hline $\begin{array}{l}\text { Korr. } \\
\mathrm{R}^{2}\end{array}$ & 0,46 & 0,44 & 0,41 & 0,47 & 0,40 & 0,45 & 0,37 & 0,47 \\
\hline $\mathrm{N}$ & 25 & 25 & 26 & 26 & 26 & 26 & 26 & 26 \\
\hline
\end{tabular}

Abhängige Variable: Polizeiexekutivbeamte in Städten über 50.000 Einwohnern auf 10.000 Einwohner, Ende 1907.

Alle Variablen logarithmiert, Regressionskoeffizienten mit Signifikanzniveau $(* * *=1 \%$ Niveau; $* *=5 \%$-Niveau; $*=10 \%$-Niveau), T-Werte in Klammern; P-Wert des F-Tests insignifikant (OLS 1/OLS4: 0,12; WLS 1/WLS 4: 0,57), daher Ausschluss insignifikanter Variablen möglich. Quellen: siehe Tab. 1.

Tab. 2: Alternative Erklärungen unterschiedlicher Polizeidichte

chen. Jedes unnötige Verweilen auf der Fahrbahn wurde verboten, der Fahrdamm sollte in beschleunigtem Schritt überquert werden. ${ }^{22}$

Nachdem der Kraftfahrzeugverkehr in der Nachkriegszeit schrittweise wieder freigegeben worden war, zeigte sich die wachsende Anerkennung des Autos in der Anhebung zulässiger Höchstgeschwindigkeiten, in Erleichterungen bei der Zulassung von Signalinstrumenten und Scheinwerfern und in der Freistellung der Kleinkrafträder von Kennzeichnungs- und Führerscheinpflicht. 1924 wurde der Beirat für das Kraftfahrwesen eingerichtet, in dem sich Behördenvertreter, Automobil-Clubs, Industrieverbände, Wege-

22 Vgl. Berliner Straßen-Polizeiverordnung vom 25. Januar 1917 und Polizeiliche Bekanntmachung zu den in der Straßen-Polizeiverordnung enthaltenden Vorschriften über Ankündigungsmittel vom 25. Januar 1917 sowie Polizeiliche Bekanntmachung über den Fußgänger-Verkehr vom 25. Januar 1917, Berlin 1917, S. 18-88. 
baupflichtige und Arbeitnehmervertreter über ihre unterschiedlichen Standpunkte zu strittigen Fragen des Kraftverkehrs austauschten. ${ }^{23}$ Unter Mitwirkung des Beirats entstand 1925 die Verordnung über Kraftfahrzeugverkehr, die entscheidende Beschränkungen der ortspolizeilichen Befugnisse mit sich brachte. Durchgangsstraßen konnten nur noch durch die oberste Landesbehörde gesperrt werden; für eine Begrenzung der zulässigen Pkw-Geschwindigkeit unter $30 \mathrm{~km} / \mathrm{h}$ wurde die Zustimmung der höheren Verwaltungsbehörde notwendig..$^{24}$ Das 1927 gegründete Polizeiinstitut für Verkehr und Technik, die Kraftverkehrsabteilung im Reichsverkehrsministerium und die Landesinnenministerien beobachteten regelmäßig ausländische Verkehrsentwicklungen, wobei den Vereinigten Staaten und insbesondere der Stadt New York eine Vorbildfunktion zukam. Autofreundliche Entscheidungen wurden in den 1920er Jahren vor allem durch diesen Einfluss technokratischer Leitbilder begünstigt. In den Ministerien trafen Interessenvertreter nun auf kompetente Ansprechpartner, die sich den Erfordernissen des wachsenden Verkehrs nicht verschlossen. Schließlich war der Krieg nach Ansicht vieler Zeitgenossen auch aufgrund zögerlicher Motorisierung verloren worden. ${ }^{25}$

Im Überwachungsalltag setzten sich strengere Anforderungen an das Vorgehen der Polizeibeamten durch. Zunächst war die Zeitnahme mit nur einer Stoppuhr zwar noch zulässig, wurde von den Gerichten aber immer öfter als unzureichend bewertet. In einem unabhängigen Gutachten des Dampfkessel-Überwachungs-Vereins Dortmund von 1924 wurde festgestellt, dass das Abstoppverfahren nur dann einigermaßen zuverlässig wäre, wenn die Stoppstrecke mindestens 300 Meter betrage und zwei Beamte mit Stoppuhren daran teilnähmen. In Berlin wurden bei Handstoppuhren Schätzungsfehler bis zu

23 Die Mitglieder des Beirats wurden vom Verkehrsminister ernannt und waren ehrenamtlich tätig. Hauptaufgabe des Beirats war es, zu wichtigen Fragen des Kraftfahrwesens Gutachten als Grundlage für die Gesetzgebung zu erstellen. Der Beirat ergänzte die Arbeit des Reichsausschusses für das Kraftfahrwesen und stand auch den Ländern in Verkehrsfragen beratend zur Verfügung. Vgl. Bundesarchiv, R 3001, Nr. 7076, Blatt 8, Verordnung über den Beirat für das Kraftfahrwesen, 5. 2. 1924; Martin Isaac, Kommentar zum Automobilgesetz, 2. Aufl. Berlin 1931, S. 708; Hans Baumann (Hg.), Deutsches Verkehrsbuch, Berlin 1931, S. 408; Fritz Müller, Die gesetzliche Neuregelung des Kraftfahrzeugverkehrs, in: C. Sperling u. E. Valentin (Hg.), Jahrbuch des Reichsverbandes der Automobilindustrie 1, Berlin 1925, S. 77-97, S. 86.

24 Vgl. Verordnung über Kfz-Verkehr, 5. 12. 1925, RGBL, 1925, S. 439, in: Pflug u. Bapst, S. $32 \mathrm{ff}$.

25 Vgl. Uwe Grandke, Verkehrsregelung und Verkehrserziehung als bewußte Modernisierung: Das Vorbild Amerika, in: Strassenverkehr und Gesellschaft 1918 bis 1933. Tagung zur Sonderausstellung „Tanz auf dem Vulkan - Die goldenen 20er in Bildern, Szenen und Objekten“, Landesmuseum für Technik und Arbeit, LTA-Forschung, H. 20, Mannheim 1995, S. 5-16; Hans-Peter Sang, Technik und Staat in der Wilhelminischen Zeit und der Weimarer Republik, in: ders. u. A. Herrmann (Hg.), Technik und Staat, Technik und Kultur, Bd. 9, Düsseldorf 1992, S. 97-119. 
fünf Prozent festgestellt, Autofahrer daher seit 1924 - bei einer zulässigen Höchstgeschwindigkeit von $30 \mathrm{~km} / \mathrm{h}$ - erst ab $40 \mathrm{~km} / \mathrm{h}$ angezeigt. ${ }^{26}$

Die Einstellung von Polizei und Behörden zum Kraftfahrzeugverkehr änderte sich auch, da sie zunehmend selbst das Auto nutzten. Dies war durchaus im Sinne der zuständigen Ministerialbürokratie, die der Verkehrspolizei eine den Kraftverkehr fördernde Funktion zuwies und an der Vereinheitlichung der zahlreichen Polizeiverordnungen arbeitete. Ab 1930 eingesetzte motorisierte Landstraßenpolizeistreifen sollten weniger Verkehrsverstöße der Autofahrer anzeigen als vielmehr ermahnend wirken. ${ }^{27}$ Doch während übergeordnete Behörden die Bedeutung des Kraftfahrzeugs für die wirtschaftliche Entwicklung erkannten und bemüht waren, hemmende Einflüsse zurückzudrängen, gingen örtliche Polizeikräfte weiterhin rigoros gegen Autofahrer vor. Durch mehrere Erlasse wies der preußische Innenminister die nachgeordneten Behörden daher in den 1920er Jahren an, jede schikanöse Anwendung polizeilicher Vorschriften zu unterlassen. 1928 wurden schließlich Richtlinien über den Erlass polizeilicher Strafverfügungen aufgestellt, die bemerkten, dass die Ortspolizeibehörden immer noch zu oft und zu hoch straften. Bei erstmaligen Übertretungen wäre, sofern es sich nicht um grobe Fahrlässigkeiten oder mutwillige Gefährdungen handelte, von einer Strafe abzusehen und stattdessen mit taktvollen Verwarnungen zu reagieren. Als selbst der preußische Ministerpräsident in eine Autofalle geriet und sich unter Berufung auf moderne Verkehrserfordernisse über das erhaltene Strafmandat beschwerte, wurde die wachsende behördliche Anerkennung des Kraftfahrzeugs offenbar. ${ }^{28}$

Bestraften viele Lokalbehörden weiterhin unnachsichtig schnellfahrende Autofahrer, mussten sie sich nun zunehmend bei vorgesetzten Stellen wegen vermeintlicher Verkehrsfeindlichkeit rechtfertigen. Nachgeordnete Stellen machten entgegen den ergangenen Weisungen oft keine Unterschiede zwischen

26 Vgl. Das Motorfahrzeug 1, 1924, Nr. 16/17, S. 9; Der Motorwagen, 1922, S. 626; 1924, S. 604; Autorechtliche Rundschau, 1929, Nr. 4, S. 4f.; Fritz Müller, Juristisches Automobil-Lexikon nebst Anhang, enthaltend das Automobilgesetz und die Verordnung über den Kraftfahrzeugverkehr, Berlin 1926, S. 163.

27 Modernität bedeutete für die Polizei in den 1920er Jahren vor allem den Einsatz moderner Technik und verkehrspolizeiliche Schulung. Der Einsatz des Autos erhöhte dabei auch die Flexibilität der Ordnungskräfte bei den zahlreichen Demonstrationen. Vgl. Richard Bessel, Militarisierung und Modernisierung: Polizeiliches Handeln in der Weimarer Republik, in: Alf Lüdtke (Hg.), „Sicherheit“ und „Wohlfahrt“. Polizei, Gesellschaft und Herrschaft im 19. und 20. Jahrhundert, Frankfurt a.M. 1992, S. 323-343, S. $327 f$.

28 Vgl. GStA PK I. HA, Rep. 77, tit. 1328, Nr. 32. spez., Bd. 4, Preussischer Ministerpräsident an Minister des Innern, 6. 4. 1927; GStA PK I. HA, Rep. 77, tit. 1328, Nr. 32gen, Preussischer Innenminister an Polizeipräsident Berlin, Regierungspräsidenten u. Oberpräsidenten, 20. 1. 1921; Kraft und Verkehr 2, 1926, Nr. 1-2, S. 1-4; Allgemeine Automobil Zeitung 30, 1929, Nr. 3, S. 9-11; 1930, Nr. 14, S. 13-16; GStA PK I. HA, Rep 77, tit. 1328, Nr. 32. spez., Bd 3, Regierungspräsident Arnsberg an preussischen Innenminister, 22. 11. 1926; Verkehrswarte, 1929, Nr. 3, S. 49f. 
Formaldelikten und tatsächlichen Verkehrsgefährdungen. In der Beurteilung der Handlungsweise von Polizeibeamten ist aber auch zu berücksichtigen, dass das Beförderungssystem den Schutzmann dazu veranlasste, möglichst viele Anzeigen zu schreiben, anstatt mit Ermahnungen eine Besserung der Verhältnisse zu erreichen. Damit trafen in der polizeilichen Observanz über den Autoverkehr nicht nur unterschiedliche Sozialschichten, sondern auch gegensätzliche Interessenlagen aufeinander. Das Auftreten der Schutzleute erzeugte bei den Autofahrern nicht das nötige Einverständnis im Straßenverkehr sondern förderte einen Konfrontationskurs. ${ }^{29}$

Die veränderte Sichtweise auf die Strafverfügungen schlug sich Ende der 1920er Jahre schließlich aber doch im statistischen Material nieder. Während 1925 noch 52.550 Strafmandate in Berlin verteilt wurden, sank deren Zahl bis 1930 auf 28.940. Trotzdem sah der ADAC weiterhin „die Demoralisierung des Verhältnisses weiter Bürgerkreise zur Obrigkeit" in der Polizeipraxis begründet. Der Club verteilte an über 150 Polizeidirektionen vorgedruckte Verwarnungszettel, die den Autofahrern Strafbefehle ersparen sollten. Schließlich konnte festgestellt werden, dass 90 Prozent der Autofallen verschwunden waren. Nur noch vereinzelt wurde moniert, dass Amtsvorsteher aus „Autohass“ Fallen betrieben, die geeignet wären, das Rechts- und Staatsbewusstsein der Autofahrer zu untergraben. Den Geschwindigkeitsbefürchtungen ländlicher Bevölkerungskreise wurde dagegen im „Zeitalter des Verkehrs" kaum Rechnung getragen. Auf Landstraßen galt weiterhin kein Tempolimit. Eine Geschwindigkeit von 60 bis $70 \mathrm{~km} / \mathrm{h}$ tolerierten die Aufsichtsorgane dabei als nicht verkehrsgefährdend. 1931 wurde in Preußen schließlich die Chausseepolizei in Ortspolizeibezirken unter 5000 Einwohnern generell den Kreispolizeibehörden (Landratsämtern) unterstellt, um endgültig die ministerielle Linie einer liberalen Fallenpolitik durchzusetzen. ${ }^{30}$

\section{Verkehrspolizisten in Werbeanzeigen aus der Motorpresse}

Als Vertreter der staatlichen Autorität traten Dorfpolizisten und Landgendarmen in einen Kontakt mit den großbürgerlichen Autofahrern, der von letzteren oft als Konfrontation empfunden wurde. Klagen über die Auto- und Verkehrsfeindlichkeit subalterner Beamter sind auch für die 1920er Jahre nachweisbar, traten aber in der Weimarer Republik zunehmend in den Hintergrund, um der Einsicht in die Notwendigkeit einer bedarfsgerechten Verkehrsregelung Platz zu machen. Diese Entwicklung lässt sich auch an der

29 Vgl. GStA PK I. HA, Rep. 77, tit 1328, Nr. 41, Bd 1, Verkehrswacht Ostpreußen an preussischen Innenminister, 25. 10. 1928.

30 Vgl. ADAC-Sport, 1926, Nr. 49, S. 6; Allgemeine Automobil Zeitung 27, 1926, Nr. 18, S. 24; 29, 1928, Nr. 52, S. 11f.; 30, 1929, Nr. 2, S. 11f., Nr. 4, S. 11ff.; 32, 1931, Nr. 33, S. 5, Nr. 51/52, S. 6; ADAC-Motorwelt, 1930, Nr. 40, S. 26; P. Weitz, Auto-Rechts-Taschenbuch für Kraftfahrer, Berlin 1925, S. 28. 


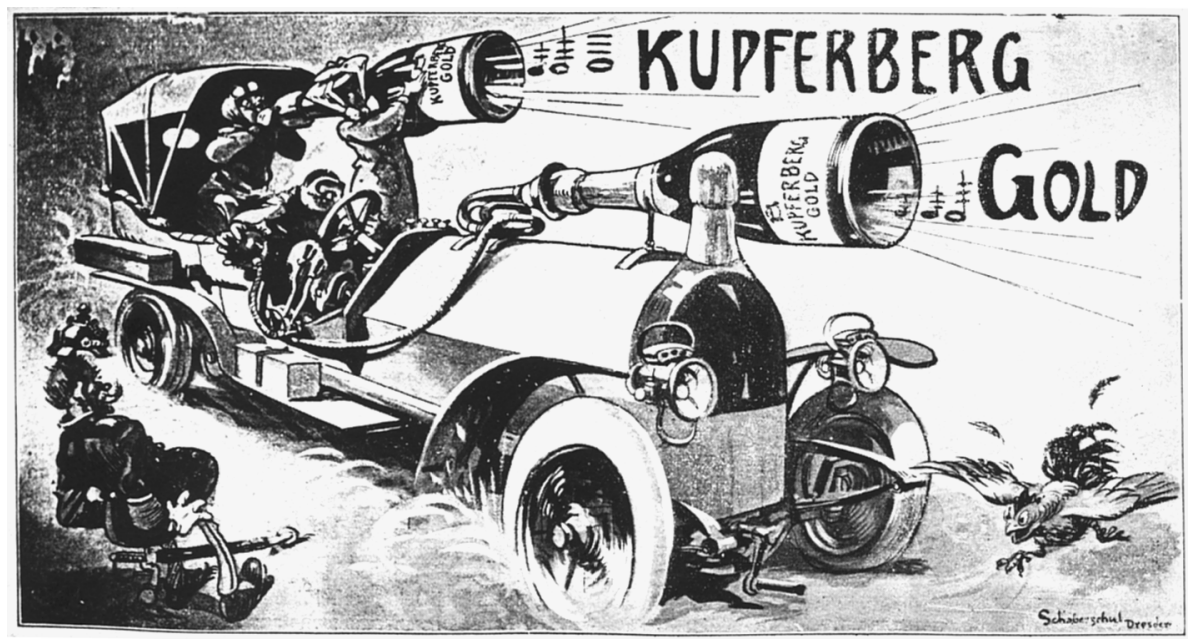

Abb. 1: Kupferberg-Werbung, 1906 (Quelle: Allgemeine Automobil Zeitung 7, 1906, Nr. 44, S. 85)

veränderten Darstellung der Polizisten in automobilistischen Werbeanzeigen beobachten. ${ }^{31}$

Den Konflikt zwischen Autofahrern und Ordnungskräften verschärfte, dass in der Kaiserzeit viele Autofahrer erstmals als Verkehrssünder mit dem Gesetz in Konflikt geraten sein dürften, da sie Schichten entstammten, die normalerweise nicht in verstärktem Maß strafrechtlicher Verfolgung ausgesetzt waren. Das Verhalten der oft dem ländlichen Milieu zugehörigen Ordnungshüter wurde daher von den autofahrenden Führungsschichten als beleidigende Zumutung verstanden, obwohl es lediglich galt, den gesetzlichen Vorschriften Geltung zu verschaffen. Aufgrund ihrer sozialen Herkunft sahen viele Dorfpolizisten ihre vordringlichste Aufgabe eher im Schutz der ländlichen Bevölkerung als in der Förderung des Verkehrslebens. Scharfe Verkehrskontrollen und das Anzeigen vermeintlicher Gesetzesübertretungen waren Möglichkeiten, den ungeliebten Städtern mit Mitteln des Gesetzes den

31 Im Gegensatz zu den Inseraten der Automobilhersteller, die nach anfänglicher Konzentration auf den Informationsgehalt bald vor allem die unbewussten Wünsche der potentiellen Käufer ansprachen, fanden sich in den Annoncen der Zulieferer versteckte Konnotationen in weit geringerem Maße. Zur Automobilwerbung und den darin transportierten Leitbildern der Mobilität vgl. Peter Borscheid, Autowerbung in Deutschland 1886-1945, in: H. Pohl (Hg.), Traditionspflege in der Automobilindustrie. Stuttgarter Tage zur Automobil- und Unternehmensgeschichte, Stuttgart 1991, S. 61-103; A. v. Pelser u. R. Scholze (Hg.), Faszination Auto. Autowerbung von der Kaiserzeit bis heute, Deutsches Werbemuseum e.V., Berlin 1994; Angela Zatsch, Reich, schnell, mobil: Automobilwerbung zu Beginn des 20. Jahrhunderts, in: P. Borscheid u. C. Wischermann (Hg.), Bilderwelt des Alltags, Werbung in der Konsumgesellschaft des 19. und 20. Jahrhunderts, Festschrift für Hans Jürgen Teuteberg, Stuttgart 1995, S. 282-293. 
Unwillen der Landbevölkerung gegen rücksichtslose Autofahrer zu verdeutlichen. Dementsprechend respektlos wurde mit den Verkehrspolizisten in den Werbeannoncen aus der Motorpresse umgegangen. Die Beamten gab man darin zunächst auch der Lächerlichkeit preis, um die Machtlosigkeit der Autofahrer gegenüber der Autofeindlichkeit lokaler Autoritäten zu kompensieren.

In der Kupferberg-Werbung von 1906 (s. Abb. 1, S. 119) bricht das Automobil, dessen Kühler und Hupe aus Sektflaschen bestehen, Fanfaren schmetternd in die ländliche Welt ein. Der Polizist reagiert ähnlich erschrocken auf die Ruhestörung wie das Federvieh. Die Pickelhaube wird beim überraschenden Auftauchen der Automobilisten in die Höhe gehoben, der Säbel ängstlich zwischen den Beinen eingeklemmt. Das vielfach als Belästigung empfundene, unablässige Hupen der Autos wird hier persifliert, wobei die Karikatur einen gewissen Stolz nicht verhehlt, dass der Autofahrer damit das Mittel besitzt, um Angstreaktionen hervorzurufen. Interessant ist dabei auch die Verbindung von Motorsport und Alkoholkonsum. Der Genuss des beworbenen Luxusproduktes Sekt, das der Warenwelt und den Konsumgewohnheiten der avisierten Zielgruppe voll entspricht, war in den Anfangsjahren des Automobilismus nicht gesetzlich verboten und keineswegs verpönt, versprach man sich doch eine belebende und konzentrationsfördernde Wirkung mäßigen Alkoholkonsums auf die Fahrfertigkeiten. Die Automobil-Clubs bekämpften zwar den Alkoholmissbrauch der Chauffeure, Forderungen nach einem absoluten Alkoholverbot für Autofahrer wurden von den Herrenfahrern aber stets strikt zurückgewiesen. Erst 1925 fragte der Reichsverkehrsminister beim Justizministerium an, ob rechtliche Bedenken beständen, eine Strafbestimmung in das Kraftfahrzeuggesetz aufzunehmen, die das Fahren in angetrunkenem Zustand generell unter Strafe stellte. Eine derartige Bestimmung war für Fuhrwerkslenker bereits in einigen Oberpräsidial-Polizeiverordnungen enthalten. ${ }^{32}$

Das lautstarke Hupen wird in der Autovox-Werbung (s. Abb. 2) auf ähnliche Weise thematisiert: Auch hier flieht Federvieh vor dem herannahenden Auto, auch hier fliegt die Pickelhaube des Polizisten in die Luft. Die „lauteste und beste Hupe der Gegenwart" hat aber zusätzlich den Vorzug, dass sie so laut ist, dass dem Beamten vor Schrecken der Notizblock aus der Hand fällt und er sich eher die Ohren zuhält, als dass er ein Strafmandat ausstellt. Das unablässige Hupen der Autos wirkte besonders ,,aufreizend“ auf die Nerven der Unmotorisierten, da es vom Publikum als Hinweis auf vermeintliche Geschwindigkeitsübertretungen interpretiert werden konnte und der Bevölkerung den Eindruck vermittelte, die Autofahrer wollten ihren Anspruch auf eine Vorrangstellung auch akustisch untermauern. Konfliktverschärfend kam

32 Vgl. Das Motorrad 4, 1906, Nr. 13, S. 228f.; Allgemeine Automobil Zeitung 8, 1907, Nr. 46, S. 52; Zeitschrift des Mitteleuropäischen Motorwagen-Verein 3, 1904, S. 207; Bundesarchiv, R 3001, Nr. 7076, Blatt 89, Reichsverkehrsminister an Reichsminister der Justiz, 3. 9. 1925. 


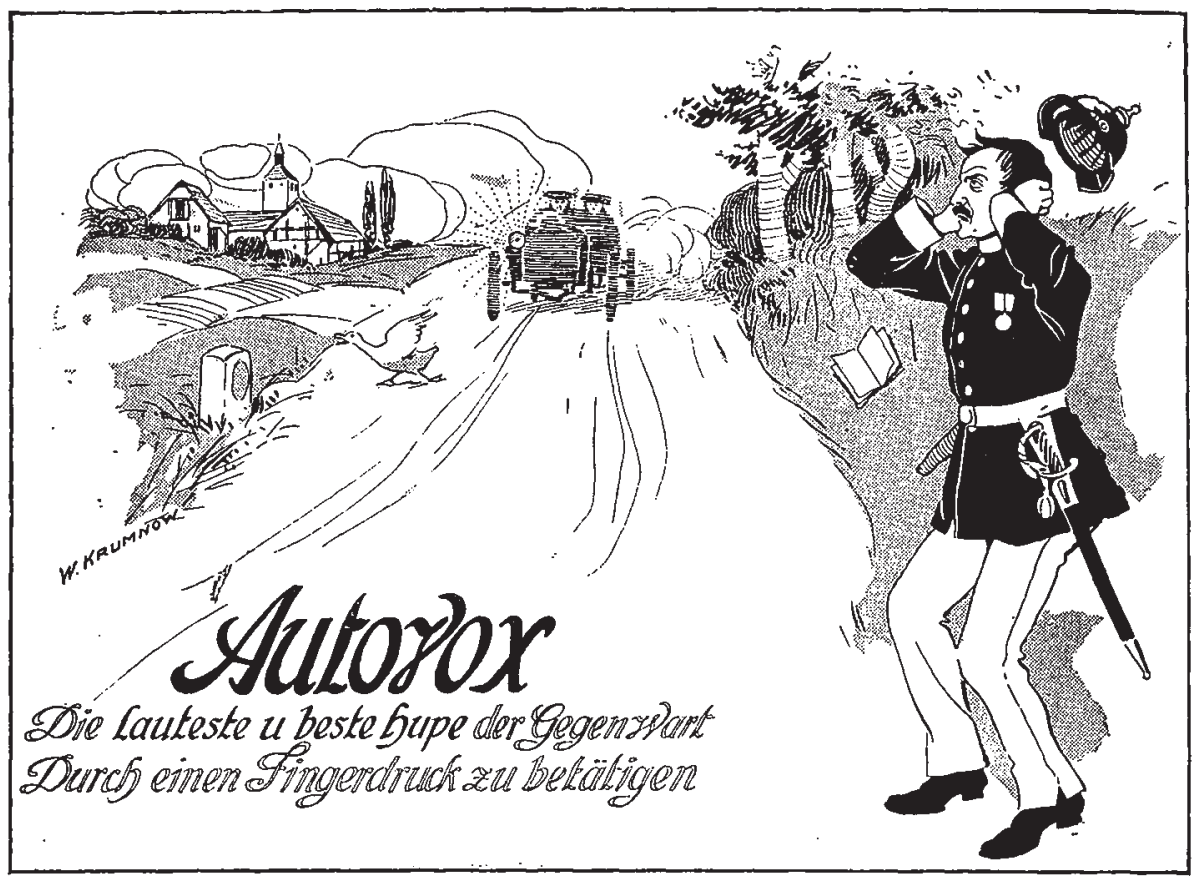

Abb. 2: Autovox-Werbung, 1909 (Quelle: Allgemeine Automobil Zeitung 10, 1909, Nr. 25, S. 30)

hinzu, dass nach Eingaben der Signalinstrumente-Industrie der Einsatz von Akkordhupen stillschweigend geduldet wurde. Allerdings befanden sich die Automobilisten hier in einem unlösbaren Dilemma, da das Hupen vielerorts vorgeschrieben war und sie Gefahr liefen, Strafmandate zu erhalten, wenn sie sich publikumsschonender verhielten. So konnte das Unterlassen nächtlichen Hupens in Wohngebieten ebenso zu Strafmandaten führen wie das übermäßige Hupen, das als ,grober Unfug“ und nächtliche Ruhestörung bestraft wurde. Wegen dieses Ermessensspielraums konnte es angesichts der verbreiteten autokritischen Grundhaltung in jedem Fall zur Verfolgung des Autofahrers kommen. ${ }^{33}$

Auch Käufer der Excelsior-Pneumatics (s. Abb. 3, S. 122) mussten Strafmandate in Kauf nehmen. Deren Höhe konnte die Genugtuung über die mit den neuen Reifen erzielten Geschwindigkeiten jedoch kaum trüben. Wer sich mit dem Luxusgefährt Auto fortbewegte, kalkulierte Strafmandate in seiner Betriebskostenrechnung ein. Der durch sein Fahrrad in der Techniknutzung

33 Sachsen wandte sich 1907 unter Verweis auf die Belästigung der Wohnbevölkerung erfolglos gegen eine Zulassung von Akkordhupen. Vgl. Bundesarchiv, R 1501, Nr. 13968, Königlich Sächsischer Minister der auswärtigen Angelegenheiten an Reichsamt des Innern, 16. 10. 1907; Automobil-Welt 1, 1903, S. 75f., S. 134f., 9, 1911, Nr. 104, S. 1; Allgemeine Automobil Zeitung 10, 1909, Nr. 6, S. 31f. 


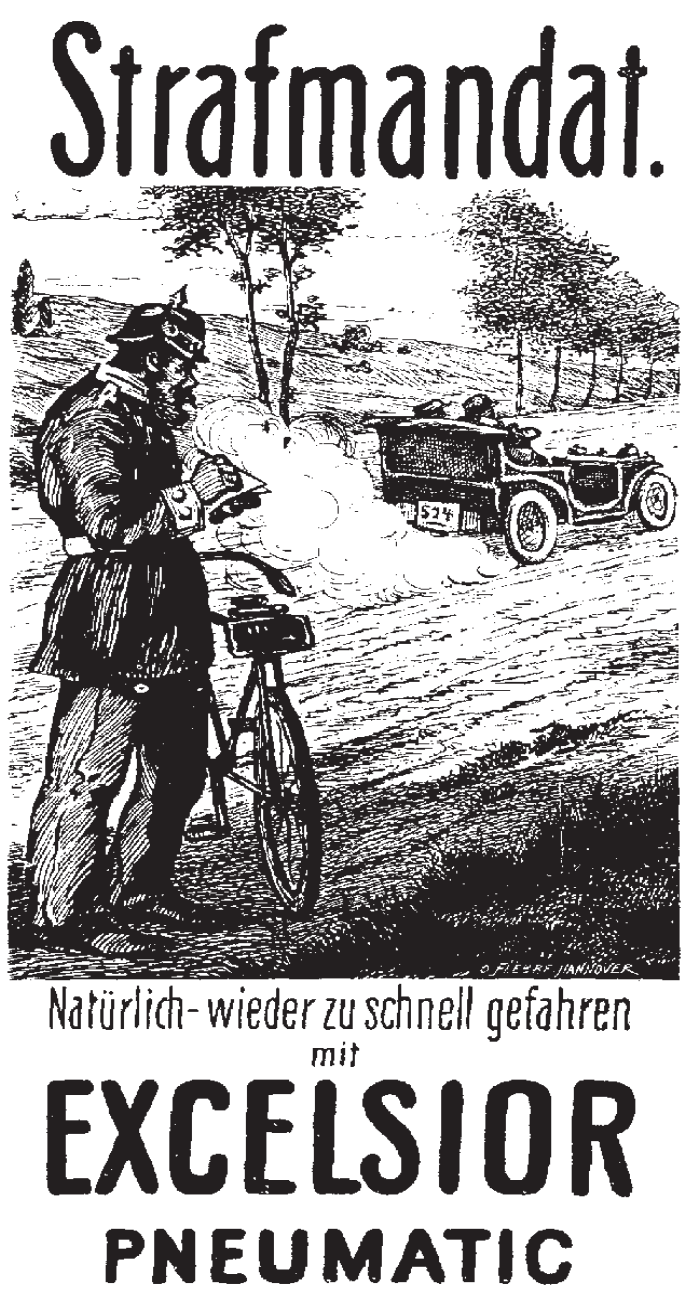

Abb. 3: Excelsior-Werbung, 1908 (Quelle: Allgemeine Automobil Zeitung 9, 1908, Nr. 12, S. 71)

sozial deklassierte Polizist wird dagegen in Staub gehüllt. Auf ein weit verbreitetes Überlegenheitsgefühl aufbauend, kann die Werbeannonce das Erhalten von Strafmandaten als für große Teile der Autofahrergemeinde schon fast zum ,guten Ton“ gehörig darstellen. Die Darstellung des fahrradfahrenden Polizisten verweist darauf, dass die Ordnungskräfte dem steigenden Verkehrsaufkommen ausstattungs- und ausbildungstechnisch zunächst nicht gewachsen waren. Bestrebungen, die Polizeibeamten dem Stand der Technik gemäß auszurüsten, stießen vielerorts auf finanziell bedingte Widerstände. Aber auch das Festhalten an traditionellen Ordnungsvorstellungen spielte eine Rolle, da selbst der Einsatz privater Motorräder zur Verkehrsüberwachung den Gendar- 


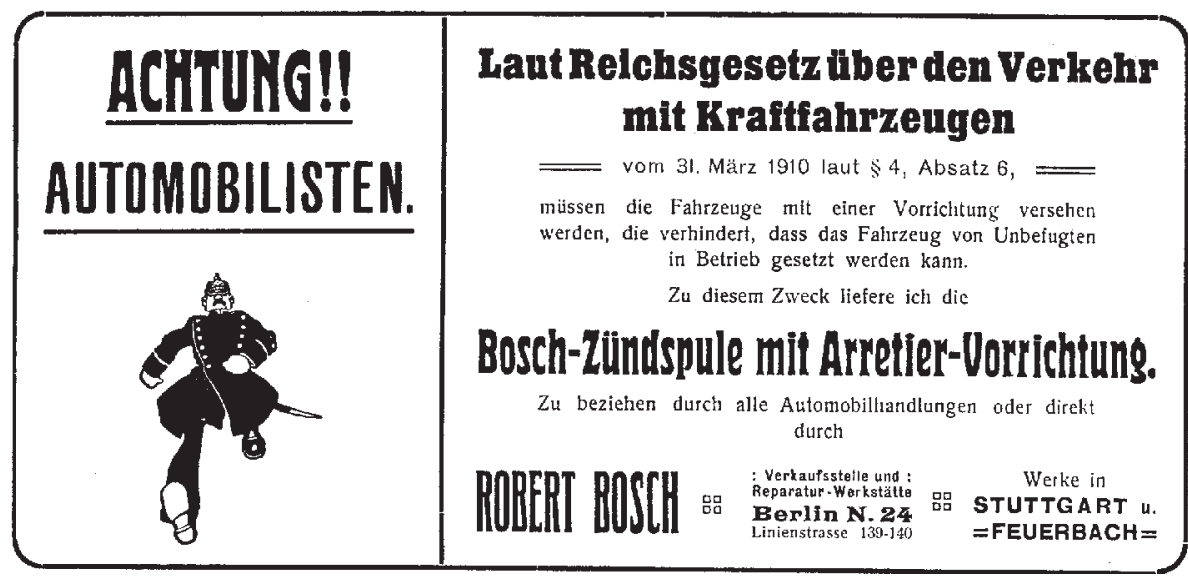

Abb. 4: Bosch-Werbung 1910 (Quelle: Allgemeine Automobil Zeitung 11, 1910, Nr. 21, S. 4)

men verwehrt blieb. Da in manchen Gegenden die Straßenverhältnisse durch Raserei unhaltbar zu werden drohten, setzte sich schließlich die Einsicht durch, die Polizeibeamten wenigstens mit modernen Stoppuhren auszustatten. ${ }^{34}$

Besonders eindringlich wirkt die Darstellung des Polizeibeamten in der Bosch-Werbung von 1910 (s. Abb. 4). Im Laufschritt kommt der Ordnungshüter mit Helm und Säbel ausgestattet auf den betrachtenden Autointeressenten zu. Mit einem plakativen „Achtung!“ wird bewusst an die Ikonografie von Verkehrsschildern mit Warnfunktion angeknüpft. Die Kaufaufforderung wird durch diese Form der bildlichen Darstellung und den Verweis auf die gesetzlichen Vorschriften zum Befehl. Will man keinen Ärger mit dem entschlossenen Polizisten riskieren, sollte man den Kauf der Bosch-Zündspule in Erwägung ziehen. Das in der Annonce erwähnte, 1909 verabschiedete Kraftfahrzeuggesetz stellte den Autoverkehr in Deutschland auf eine einheitliche Grundlage. Es löste zwölf preußische und 19 Polizei-Verordnungen über den Kraftverkehr der anderen Bundesstaaten ab, wodurch es zu einer gewissen Vereinheitlichung kam. Auch wenn regionale Sonderregelungen möglich blieben, hatte die vormalige Vielfalt unterschiedlicher Verordnungen für den Autofahrer eine weit größere Unsicherheit bedeutet, da zu Beginn jeder Polizeibezirk auf Einhaltung seiner lokalen Bestimmungen bestanden hatte. Ausgeführt wurde das Kraftfahrzeuggesetz durch eine Bundesratsverordnung von 1910. Von der darin vorgesehenen Möglichkeit, das Tempolimit zu erhöhen, machten zahlreiche Städte Gebrauch, da der entscheidende Vereinheitlichungsschritt ein höheres $\mathrm{Ma} ß$ an Verkehrssicherheit erwarten

34 Vgl. GStA PK I. HA, Rep. 77, tit. 1712, Nr. 16a, Chef der Landgendarmerie an U.R. der 12. Brigade (Danzig), 3. 7. 1908; GStA PK I. HA, Rep. 77, tit. 1712, Nr. 16a, Regierungspräsident Coblenz an Minister des Innern, 14. 4. 1913. 


\section{Todsicher schítz MuWag vor Strafmandaten und Unfällen
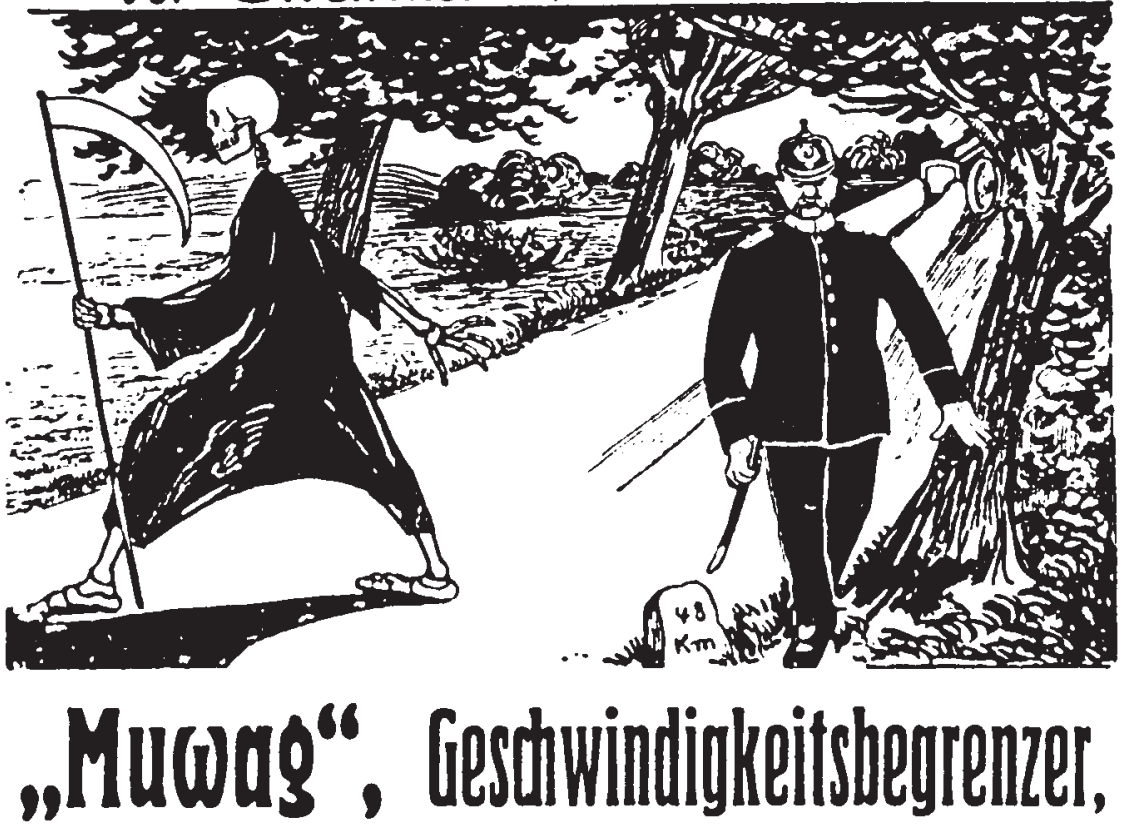 rerbunden mit Geschwindigkeitsmesser u. Rilometerzähler.}

Abb. 5: Muwag-Werbung, 1911 (Quelle: Allgemeine Automobil Zeitung 12, 1911, Nr. 8, S. 11)

ließ. In Berlin und den meisten preußischen Städten durften die Autofahrer 25 $\mathrm{km} / \mathrm{h}$ schnell fahren, während in Hamburg und München sowie in vielen anderen bayerischen Städten eine Höchstgeschwindigkeit von $20 \mathrm{~km} / \mathrm{h}$ galt. Auch die beständige Kritik an Begleiterscheinungen des Autoverkehrs hatte dazu beigetragen, dass die Grundlagen für eine moderne Straßenverkehrsordnung früh gelegt wurden. Obwohl den Kritikern des neuen Verkehrsmittels die Strafvorschriften nicht weit genug gingen, hatte das Kraftfahrzeuggesetz auf die Automobilakzeptanz eine positive Wirkung, da Sicherheitsprobleme verringert werden konnten. Die Vereinheitlichung rechtlicher Grundlagen lag auch im Interesse der Autofahrer, so dass eine grundsätzliche Zustimmung der Interessenvertreter erreicht werden konnte, zumal diese im Gesetzgebungsverfahren ihren Einfluss geltend machen konnten. ${ }^{35}$

351923 wurde die zulässige Höchstgeschwindigkeit in Berlin dem Verkehr erneut angepasst und auf 35 km/h festgesetzt. Vgl. Der Motorwagen 26, 1923, S. 318; Angela Zatsch, Staatsmacht und Motorisierung am Morgen des Automobilzeitalters, Konstanz 1993, S. 185ff.; Fraunholz (wie Anm. 3), S. $211 \mathrm{ff}$. 
Auch in der Muwag-Werbung (s. Abb. 5) wird auf die unangenehmen Begleiterscheinungen eines Kontaktes mit den Ordnungskräften angespielt. Das Strafmandat wird hier gar mit makaberem Sarkasmus einem tödlichen Unfall gleichgestellt. Der den Unfalltod symbolisierende Sensenmann und der gestreng blickende Gendarm erscheinen als verwandte Gesellen, die zum Schaden der Autofahrer die Landstraßen bevölkern. Dabei wird die Ansicht vertreten, dass die Autofahrer in ihrem eigenen Interesse die Fahrgeschwindigkeit mindern sollten, wobei sie der Muwag-Geschwindigkeitsbegrenzer unterstützen könnte. Das beworbene Produkt schütze den Automobilisten ,todsicher" vor derartigen Beeinträchtigungen der automobilen Fortbewegung. Bereits 1903 wurde in der Automobilpresse ein Geschwindigkeitsregistrator als „Selbstschutz gegen unrichtige polizeiliche Denunziationen“ empfohlen. Tachometer gehörten in den Anfangsjahren des Autos keineswegs zur Standardausstattung. Zudem arbeiteten sie ungenau, und die Aufzeichnungen der integrierten Fahrtenschreiber wurden bei Gericht oft nicht als Beweismittel anerkannt. Dennoch setzten sich Tachos allmählich als gebräuchliches Zubehör durch, da ihr Vorhandensein die Möglichkeit eröffnete, im Fall einer Gerichtsverhandlung auf ihre genaue Beobachtung zu verweisen und das Einhalten des Tempolimits zu beschwören. Neben der technischen Aufrüstung konnte auf kritischen Wegstrecken besonders vorsichtig gefahren werden, um Strafmandate zu vermeiden. Die Automobilpresse veröffentlichte zu diesem Zweck regelmäßig Warnungen vor den zahlreichen „Automobilfallen“. Dies hatte den Nebeneffekt, dass die betreffenden Orte soweit möglich von Autofahrern

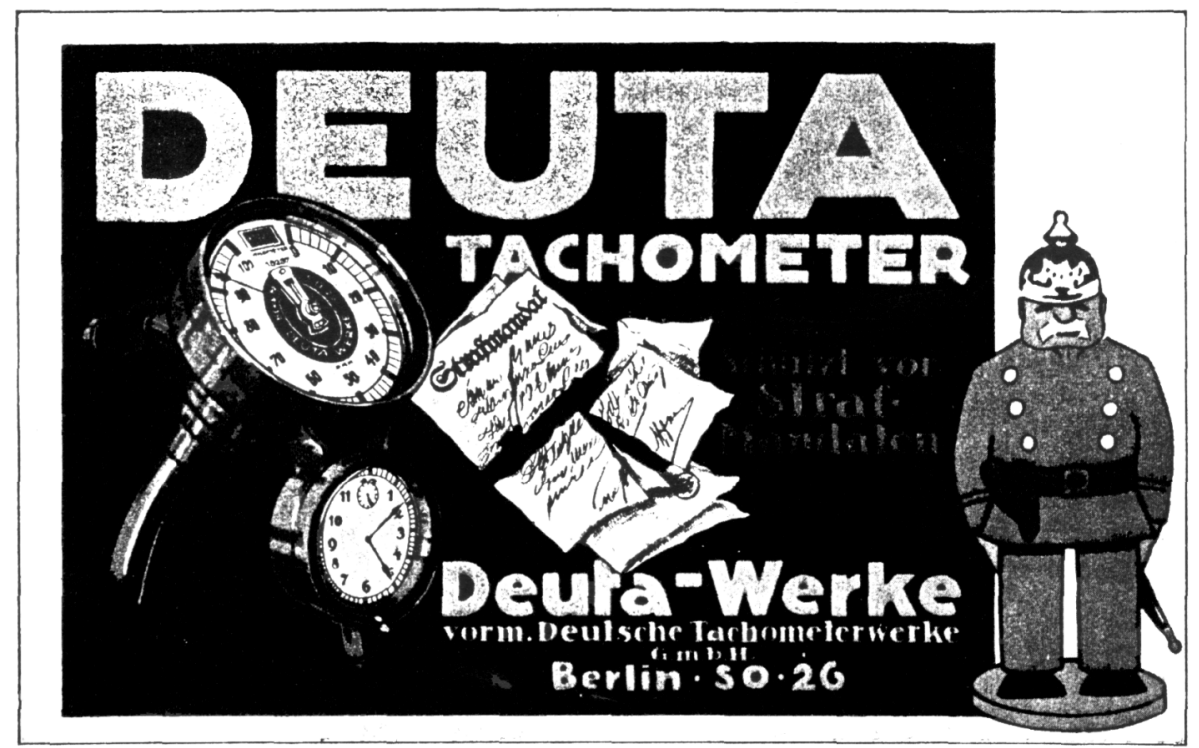

Abb. 6: Deuta-Werbung, 1913 (Quelle: Motor 1, 1913, Nr. 4, S. 128) 


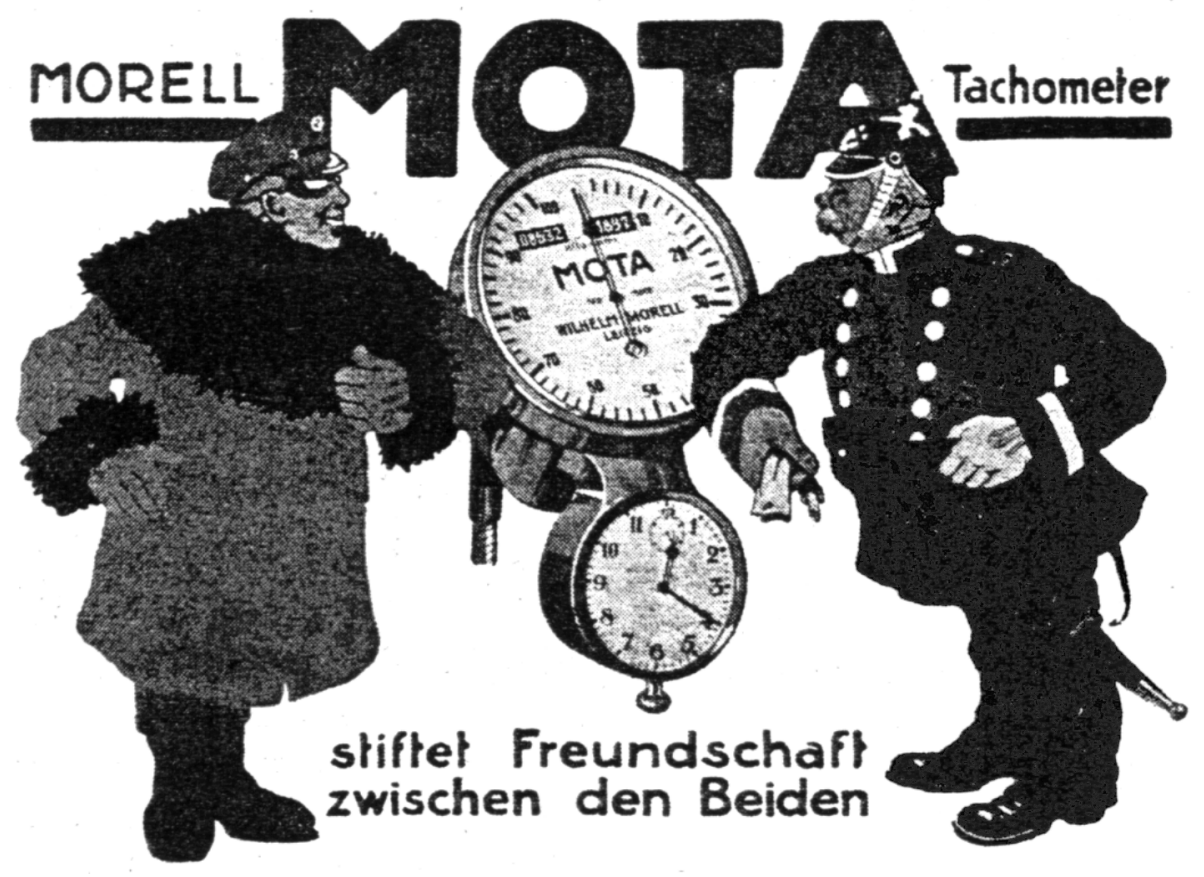

Abb. 7: Morell-Werbung, 1912 (Quelle: Der Motorwagen 15, 1912, S. 711)

gemieden wurden, wodurch wirtschaftliche Schädigungen eintreten konnten. Das einfachste Mittel, ein Strafmandat zu umgehen, bestand aber darin, falls man eine polizeiliche Überwachung rechtzeitig bemerkt hatte, innerhalb der Stoppstrecke anzuhalten. Die Berechnung einer Durchschnittsgeschwindigkeit wurde dadurch unmöglich gemacht. ${ }^{36}$

In der Deuta-Werbung von 1913 (s. Abb. 6, S. 125) sollte der Polizeibeamte lächerlich gemacht werden, indem er als harmlose Kühlerfigur dargestellt wurde. Seine statische Erscheinung bildet einen auffälligen Gegensatz zur ungehemmten Beweglichkeit der Autofahrer. Diese könnten nach dem Kauf des Tachometers etwa erhaltene Strafmandate mit gutem Gewissen zerreißen, da sie sich vor Gericht auf die durch den eingebauten Fahrtenschreiber nachgewiesenen, tatsächlich erzielten Höchstgeschwindigkeiten berufen könnten. Diese Werbeanzeige unterstellt somit, dass Strafmandate stets auf den falschen Beobachtungen der Beamten beruhten. Durch den Einbau des DeutaProdukts seien diese künftig zu tatenloser Starre verdammt. Die Verwendung

36 Der Landrat von Koblenz ging 1908 gegen eine so genannte „Straßenverbotskarte“ gerichtlich vor. Die Staatsanwaltschaft Frankfurt lehnte eine Verurteilung wegen angeblicher Beleidigung der Polizeiorgane durch als „Automobilfallen“ bezeichnete Orte jedoch ab. Vgl. Allgemeine Automobil Zeitung 7, 1906, Nr. 46, S. 92; 9, 1908, Nr. 41, S. 45f.; Zeitschrift des Mitteleuropäischen Motorwagen-Verein, 1903, Nr. 6, S. $157 \mathrm{f}$. 


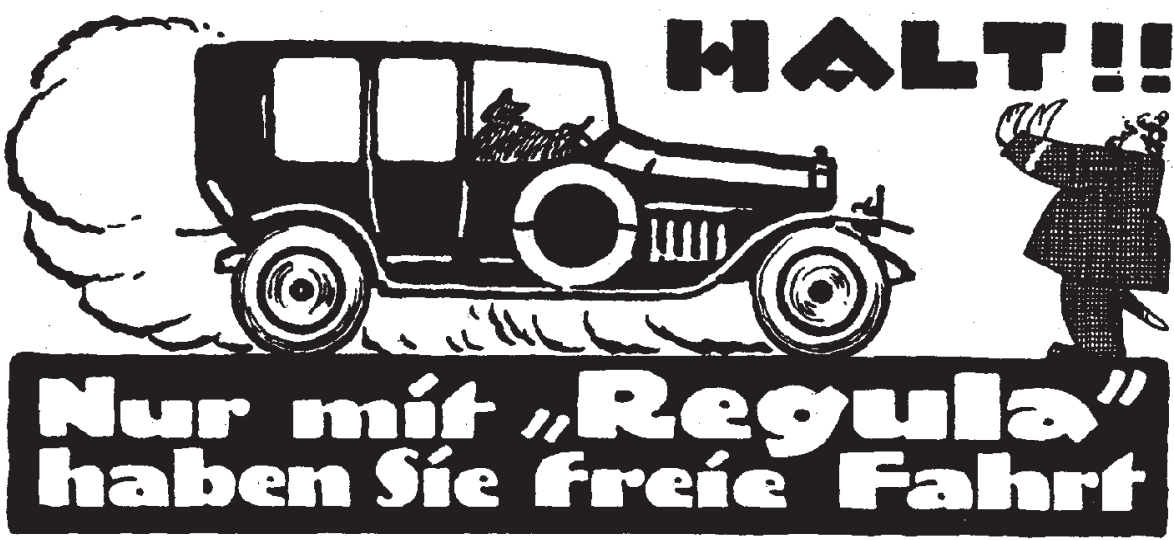

Abb. 8: Regula-Werbung, 1920 (Quelle: Allgemeine Automobil Zeitung 21, 1920, Nr. 8, S. 5)

einer Abbildung, die den Polizisten als Kühlerfigur darstellt, spielt auch auf eine aus Frankreich und Großbritannien importierte Mode an, die einige $\mathrm{Au}$ tofahrer dazu bewog, ihre Wagen mit Kühlermaskotten in Polizistenform auszustatten. 1910 kam es vor dem Königlichen Landgericht München zu einer Verhandlung wegen des derart inkriminierten Kühlerschmucks. Das Gericht sah den Tatbestand des groben Unfugs erfüllt, da die Figur ,zum Zwecke der Verhöhnung der Polizeiorgane" angebracht worden sei, und verurteilte den Autofahrer zu einer Geldstrafe von 40 Mark. Der anzeigeerstattende Schutzmann sagte bei der Verhandlung aus, dass er sich durch den Anblick der Kühlerfigur in seiner Würde verletzt fühlte und aus dem Publikum ebenfalls Missfallensäußerungen an der Figur vernommen hätte. ${ }^{37}$

Eine versöhnlichere Darstellung des Polizisten wählt dagegen die MorellWerbung von 1912 (s. Abb. 7). Die äußere Erscheinung des Schutzmanns mit Uniform, Haube, Säbel und Schnauzbart ähnelt zwar der Darstellung als Kühlerfigur, drückt hier jedoch gutmütige Behäbigkeit aus. Der Ordnungshüter zerknüllt das Strafmandat, während er sich mit dem martialisch gekleideten Automobilisten über die Vorzüge des Mota-Tachometers austauscht. Dass das beworbene Produkt Freundschaft zwischen Autofahrern und Ordnungsorganen stiften könnte, erscheint allerdings als ironisierender Euphemismus, bedenkt man das Ausmaß der Konflikte zwischen den beiden Parteien. Mit seiner Leibesfülle und den aus Pickelhaube und Säbel bestehenden Insignien wirkt der

37 Vgl. Allgemeine Automobil Zeitung 11, 1910, Nr. 40. S. 43; Nr. 49, S. 53. In einem Versandkatalog für Autozubehör wurde eine ähnliche Kühlerfigur angeboten: „Schutzmann, humoristische Kühlerfigur. Die höchst amüsante Kühlerfigur stellt einen Schutzmann dar, dessen ausgestreckte Arme durch den vorne montierten Propeller in Bewegung gesetzt werden. Höhe der Figur: $110 \mathrm{~mm}$, Armweite: $150 \mathrm{~mm}$, ganz aus Aluminium, daher geringes Gewicht. M. 23“; Sorge \& Sabeck GmbH (Hg.), SoSa-Motormaterial, Der Führer durch die internationale Zubehörindustrie, Berlin o. J. (um 1910), S. 147. 
Polizeibeamte in der Regula-Werbung von 1920 (s. Abb. 8, S. 127) dagegen - angesichts der veränderten politischen Verhältnisse - wie ein Vertreter einer überkommenen Zeit. Im Kommandoton, der gleichzeitig die Kaufaufforderung unterstreicht, stemmt er sich dem Auto, das den technischen und gesellschaftlichen Fortschritt verkörpert, entgegen.

Die durch typographische Größenunterschiede bewusst missverständlich gestaltete Aufforderung in der fünf Jahre jüngeren Sonex-Anzeige (s. Abb. 9) grenzt dabei fast an Beamtenbeleidigung, da man beim ersten Hinsehen an eine Äußerung des angesichts des gestikulierenden Polizisten entnervten Autofahrers denkt. Tatsächlich bildet sie aber eine Aussage des dargestellten Ordnungshüters ab und bezieht sich auf die Auspuffklappe des Wagens. Die Annonce reflektiert somit die Diskussionen um dieses Zubehörteil, die in den 1920er Jahren intensiv geführt wurden, und verspricht Abhilfe durch Verwendung des beworbenen Produkts. Die Darstellung des abgebildeten Polizisten ist dabei neutral, allein durch den Text erhält die Anzeige ihre Zweideutigkeit. Das Öffnen der Auspuffklappe, das den Motor von Auspuff und Schalldämpfung trennte, um eine Leistungssteigerung zu bewirken, war zunächst noch auf offener Landstraße zulässig, wurde aber wegen der damit verbundenen Geräusch- und Abgasbelästigung 1910 gänzlich verboten und streng bestraft. Da es jedoch trotzdem weiterhin zu ständigen Zuwiderhandlungen kam, wurden in den 1920er Jahren strengere Bestimmungen durchgesetzt. Bis zum 1. März 1926 mussten alle an bereits zum Verkehr zugelassenen Fahrzeugen

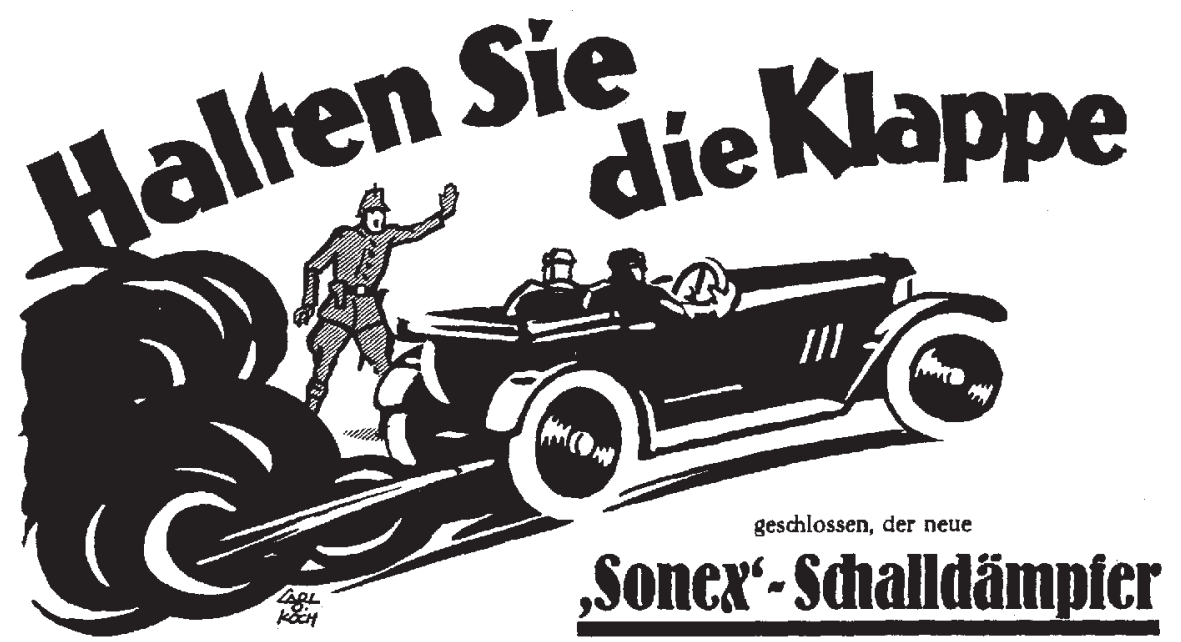

erübrigł jede Auspuffklappe, da die Gase automatisch abgesaugt und die $L$ e i 5 i $n g$ gegenūber offenem Auspuff noch erhäht wird. * Lieferbar für A u tom obile und Motorrãder

"Sonex" Apparatebau G. m. b. H. * Herford i. Westf. $\begin{array}{llllllllllllllllllllllllllll}S & p & e & z & i & a & i & f & a & b & r & i & k & f & u & r & A & u & s & p & u & f & f & f & \tilde{o} & p & f & e\end{array}$

Abb. 9: Sonex-Werbung, 1925 (Quelle: Allgemeine Automobil Zeitung 26, 1925, Nr. 5, S. 11) 


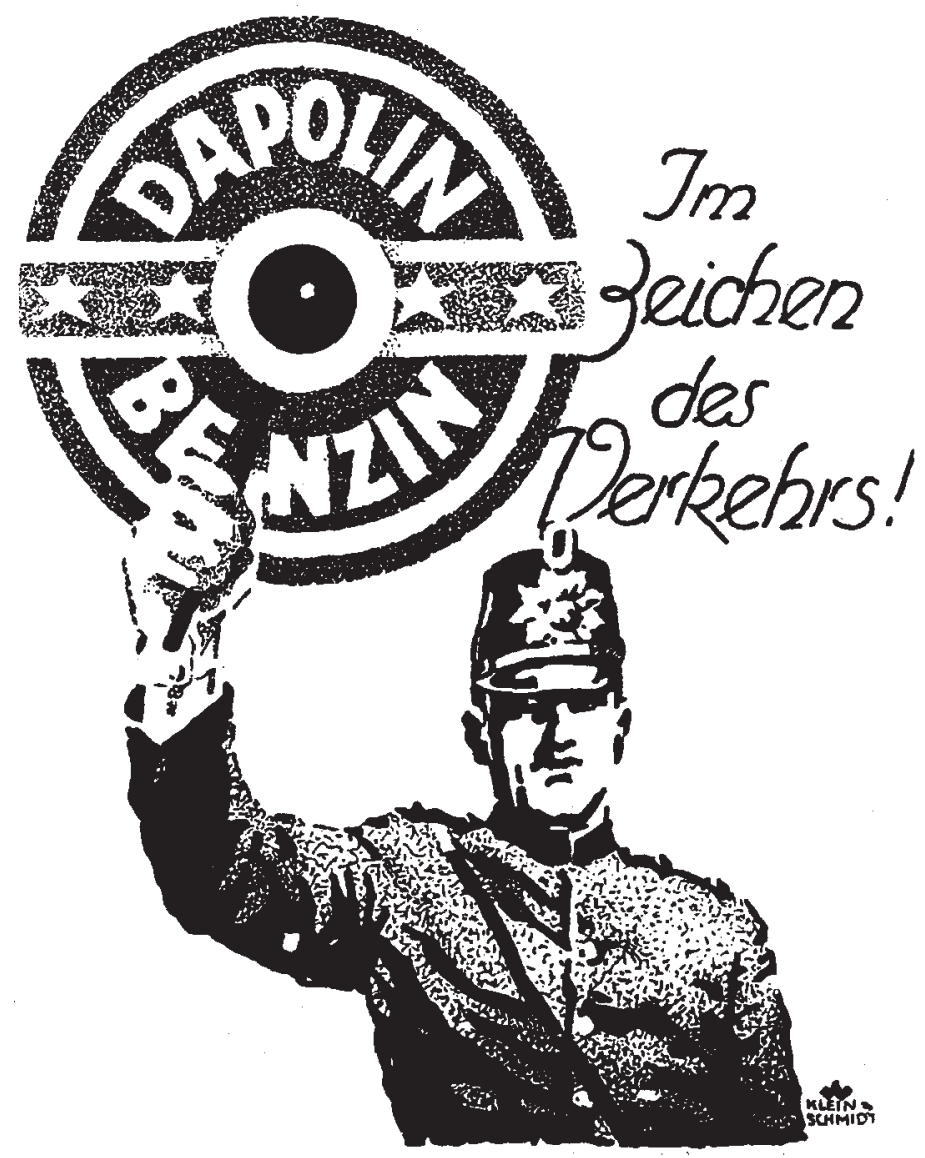

Abb. 10: Dapolin-Werbung, 1926 (Quelle: Allgemeine Automobil Zeitung 27, 1926, Nr. 22, S. 40)

angebrachten Auspuffklappen entfernt werden, so dass eine Trennung von Motor und Auspuff nicht mehr stattfinden konnte. Darüber hinaus wurde nun jegliche belästigende Rauch- und Geruchsentwicklung ausdrücklich untersagt. ${ }^{38}$

In der Dapolin-Werbung (s. Abb. 10) von 1926 wird der Verkehrspolizist dagegen bereits als vollwertiger Teil des modernen Verkehrs anerkannt. „Im Zeichen des Verkehrs" ist die Verwendung von Dapolin-Benzin empfehlenswert, gleichzeitig ist eine zeitgemäße Verkehrsregulierung durch die Ordnungskräfte notwendig geworden. Der Polizist wird nicht mehr als störendes Bei-

38 Vgl. Bundesarchiv, R 1501, Nr. 14143, Reichsverkehrsministerium, Niederschrift: Mißstände im Kraftfahrwesen, 8. 10. 1924. S. 1; Schreiben eines Bürgers an den Präsidenten des Reichsgesundheitsamtes, 13. 2. 1910, in: Franz-Josef Brüggemeier u. Michael Toyka-Seid (Hg.), Industrie-Natur, Lesebuch zur Geschichte der Umwelt im 19. Jahrhundert, Frankfurt a. M., New York 1995, S. 250f.; Allgemeine Automobil Zeitung 11, 1910 , Nr. 28, S. 51; Nr. 16, S. 50; Automobil - Motorrad - Flugwesen 8, 1926, Nr. 3, S. 54. 


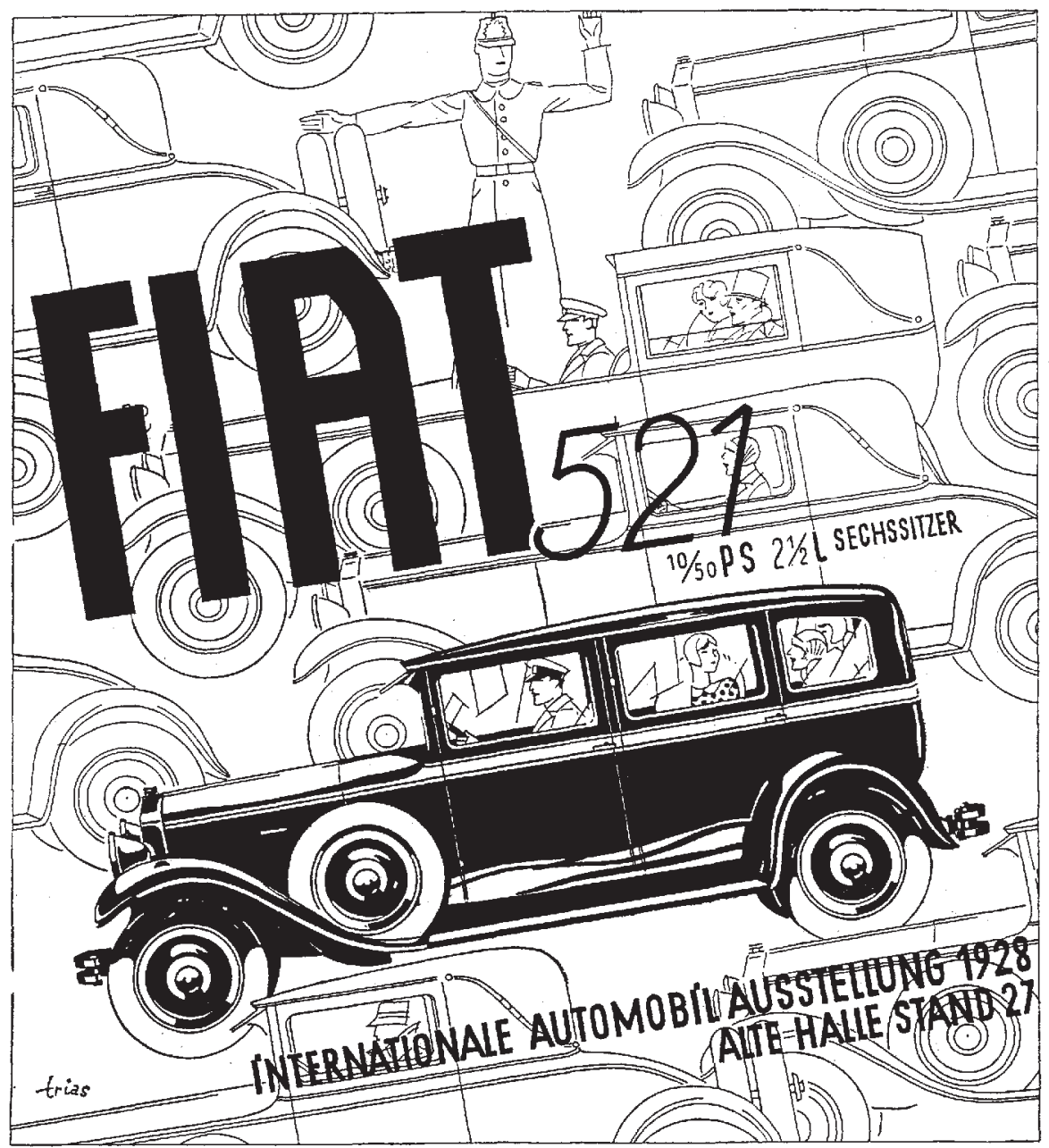

DEUTSCHE FIAT-AUTOMOBIL-VERKAUFS-A. - G. BERLIN-TEMPELHOF, INDUSTRIESTR. $35-37$ NIEDERLASSUNG: KÖLN-LINDENTHAL, DURENER STRASSE 132

SUDDEUTSCHE FIAT-AUTOMOBIL-VERKAUFS-A.-G. MUNCHEN, BERG - AM-LAIM-STRASSE 31

EIGENE VERKAUFSSTELLE: LENBACHPLATZ

Abb. 11: Fiat-Werbung, 1928 (Quelle: Allgemeine Automobil Zeitung 29, 1928, Nr. 44, S. 15)

werk oder Bedrohung empfunden, seine Arbeit ermöglicht vielmehr erst die ungehinderte Zirkulation der Automobile. Vollends in den großstädtischen Autoverkehr integriert, zeigt sich der Verkehrspolizist in der abgebildeten Fiat-Werbung (s. Abb. 11). Skizzenhaft wird er in der Vielzahl der eleganten Automobile, die ihn fast zu erdrücken drohen, sichtbar. Auf sein regulierendes Eingreifen sind die Autofahrer angewiesen, soll es nicht zu Unfällen und 
damit zum Stillstand kommen. Gleichzeitig verheißt seine Handbewegung „Freie Fahrt“. Um den modernen Verkehr zu bewältigen, benötigen die Verkehrspolizisten in gesteigertem Maße ,kühle Besonnenheit, ruhiges Blut und stete Geistesgegenwart", wie die Werbebotschaft der Dallmann-Werbung (s. Abb. 12) lehrt: Eigenschaften, die auch von den anderen Verkehrsteilnehmern gefordert werden, um im täglichen Verkehrsgewühl zu bestehen. Ein freundlicher Beamter, dessen anstrengender Beruf am Ende des untersuchten Zeitraums voll anerkannt wurde, durfte daher für ein Aufbaupräparat werben, das von Autofahrern gekauft werden sollte.

Die erläuterten Werbeanzeigen verdeutlichen, wie sich die Sicht der Industrie und damit der angesprochenen Autofahrer auf die Verkehrspolizisten mit Zunahme des motorisierten Straßenverkehrs veränderte. Wurden Polizeibeamte in der Kaiserzeit vielfach als feindlich gesinnte Staatsvertreter gese-

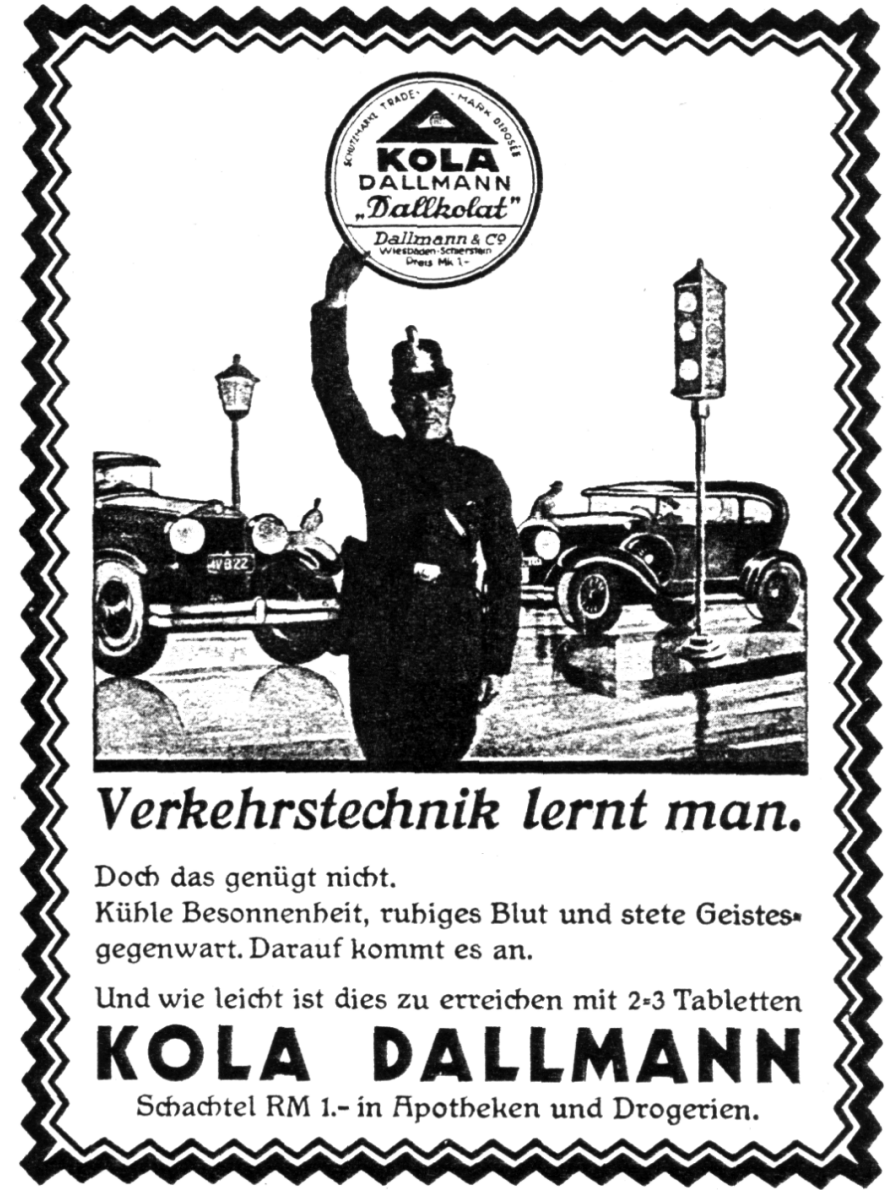

Abb. 12: Dallmann-Werbung, 1930 (Quelle: Allgemeine Automobil Zeitung 31, 1930, Nr. 25, S. 34) 
hen, die man der Lächerlichkeit preisgab, um die Machtlosigkeit der Autofahrer gegenüber staatlicher Autorität zu kompensieren, setzte sich ab Mitte der 1920er Jahre eine Sichtweise durch, die ihre Anwesenheit als Notwendigkeit interpretierte. Beigetragen haben dazu sicherlich auch die zahlreichen Weisungen übergeordneter Stellen, die wiederholt eine verkehrsfördernde Rolle der Beamten betonten und Willkürakte sowie verstecktes Protokollieren zu unterbinden suchten. Die herablassende oder karikierende Abbildung von Verkehrspolizisten, die für die Kaiserzeit typisch war, fand sich in den Werbebotschaften der Auto- und Zulieferindustrie seit der zweiten Hälfte der 1920er Jahre nicht mehr. Allenfalls in der zeitgenössischen Karikatur konnten die Polizisten noch der Lächerlichkeit preisgegeben werden. Ihre Darstellung in einer „Autofalle“ mit heruntergelassenen Hosen, die die Allgemeine Automobil Zeitung noch 1932 brachte, spielte dabei auf das von den Automobilvereinen weiterhin vehement bekämpfte, versteckte Protokollieren in Zivil an. ${ }^{39}$

\section{Die Verkehrspolizei als schlichtende Institution}

Das in seiner Pionierzeit vor allem als „Prestigesymbol“ und Sportgerät genutzte Auto diente den Besitzbürgern dazu, ihren Anspruch zu dokumentieren, ,als Herren über Raum und Zeit auch zu Herren über die soziale Ordnung zu werden. ${ }^{“ 40}$ Als öffentlich genutztes Luxusgut stellte es ein adäquates Ausdrucksmittel für den Prestigebedürfnissen entspringenden ,demonstrativen Konsum“ der „feinen Leute“ dar. ${ }^{41}$ Da neben dieser klassendifferenzierenden Wirkung des Autos erste Folgekosten der Motorisierung rasch deutlich wurden, erkannten die Behörden früh die Notwendigkeit, ,schlichtend“ mittels Kraftfahrzeuggesetzgebung in die Verkehrskonflikte einzugreifen. Eine verstärkte Polizeipräsenz kann dabei mit den erhöhten Unfallgefahren des Autoverkehrs erklärt werden. Erklärungen für die Varianz der Polizeidichte, die Schutz- und Fürsorgeaufgaben der Polizei einbeziehen, sind daher gegenüber Interpretationen, die allein eine - fraglos bedeutende - Repressionsfunktion betonen, zu favorisieren. ${ }^{42}$

Begünstigt wurde das verkehrsfeindliche Verhalten unterer Polizeibehörden durch die Rechtsunsicherheit, in der sich der frühe Automobilismus bewegte, sowie durch die nach der gesetzlichen Regelung verbleibenden Ermes-

39 Vgl. Allgemeine Automobil Zeitung 33, 1932, Nr. 39, S. 10.

40 Wolfgang Sachs, Die automobile Gesellschaft, Vom Aufstieg und Niedergang einer Utopie, in: F.-J. Brüggemeier u. Th. Rommelspacher (Hg.), Besiegte Natur. Geschichte der Umwelt im 19. und 20. Jahrhundert, München 1987, S. 106-123, S. 111.

41 Vgl. Thorstein Veblen, Theorie der feinen Leute, Eine ökonomische Untersuchung der Institutionen, Frankfurt a. M. 1986 (amerik.1899).

42 Freilich können auch „,vornehmlich unterstützende Tätigkeiten“ der Polizei, wie die Verkehrsregelung, repressive Elemente und physischen Zwang enthalten. Dieser richtete sich aber, zumindest bis zum Ersten Weltkrieg, vor allem gegen die besitzbürgerlichen Autofahrer. Vgl. Alf Lüdtke, Einleitung: „Sicherheit“ und „Wohlfahrt“, Aspekte der Polizeigeschichte, in: ders. (wie Anm. 27), S. 7-33, S. 7. 
sensspielräume. Die Pedanterie in der Auslegung von Verkehrsvorschriften auf das autoritäre Gesellschaftsverständnis der kleinbürgerlichen Polizisten zurückzuführen, wie dies viele Automobilisten taten, greift jedoch zu kurz. Vielmehr wurden die Landpolizisten zu Gegnern des Autos, indem sie den Unmut der Bevölkerung über die Zumutungen des motorisierten Straßenverkehrs aufnahmen und in ihre Handlungsmöglichkeiten übersetzten. Zudem lieferten die zahlreichen Strafmandate willkommene Zusatzeinnahmen für die durch die Erfordernisse des Straßenunterhalts erschöpften Gemeindekassen. Aufgrund ihrer sozialen Herkunft sahen viele Dorfpolizisten eher im Schutz der Bevölkerung als in der Förderung des Verkehrs ihre vordringlichste Aufgabe. Scharfe Verkehrskontrollen und das Anzeigen vermeintlicher Gesetzesübertretungen waren Möglichkeiten, den ungeliebten Städtern mit Mitteln des Gesetzes den Unwillen der Landbevölkerung gegen rücksichtslose Autofahrer zu verdeutlichen. Die schikanöse Handhabung bestehender Vorschriften brachte die autokritische Haltung der Wohnbevölkerung zum Ausdruck und setzte gewalttätige Proteste mit legalen Mitteln fort. Als drakonisch empfundene Strafen reizten die Autofahrer, so dass es zu einem Fehlverhalten kommen konnte, das über die Überschreitung der zulässigen Höchstgeschwindigkeit hinausging und bei Beamtenbeleidigungen auch Arreststrafen nach sich ziehen konnte.

Den Konflikt zwischen Autofahrern und Ordnungskräften verschärfte, dass in der Kaiserzeit viele Autofahrer erstmals als Verkehrssünder mit dem Gesetz in Konflikt gerieten, da sie Schichten entstammten, die nicht in verstärktem Maße strafrechtlicher Verfolgung ausgesetzt waren. Das Verhalten der oft dem ländlichen Milieu zugehörigen Verkehrspolizisten wurde daher von den autofahrenden Führungsschichten als beleidigende Zumutung verstanden, obwohl es lediglich galt, den gesetzlichen Vorschriften Geltung zu verschaffen. Die Motorpresse interpretierte das behördliche Verhalten zuweilen gar als ursächlich für gewalttätige Eskalationen und stellte in Bezug auf die Polizeiverhältnisse in Baden fest: „Aber es ist eine bekannte psychologische Erscheinung, dass der Eifer der Behörden, wenn er zufällig mit der Lust der Masse übereingeht, in diesen Massen zu Ausschreitungen ausartet. Interessante Fälle dieser Art finden wir in den russischen Pogroms. “43

Der erbitterte Kampf zwischen Autofahrern und Polizisten um die strikte Einhaltung von Verkehrsvorschriften wurde auch mit Bildern geführt und setzte sich in den Werbeannoncen der Zulieferindustrie als Kompensierung von Machtlosigkeit fort. Dabei zeigten sich erstaunliche Spielräume im kritischen Umgang mit den Repräsentanten des Obrigkeitsstaates. Die pejorative, mediale Verarbeitung verkehrspolizeilicher Observanz trat allerdings in der Weimarer Republik ebenso in den Hintergrund, wie die Klagen über die Auto- und Verkehrsfeindlichkeit subalterner Beamter. Stattdessen setzte sich die Einsicht in die Notwendigkeit einer bedarfsgerechten Verkehrsregelung durch. In den

43 Automobil-Welt 5, 1907, Nr. 125, S. 1. 
1920er Jahren nahmen Verkehrspolizisten auf Druck der Zentralbehörden überwiegend verkehrsfördernde Haltungen ein.

Die polizeiliche Überwachung des Autoverkehrs fügte sich in die allgemeine Tendenz zur Spezialisierung der Polizeikräfte ein und war in den 1920er Jahren mit einer verbesserten Ausbildung ebenso verbunden, wie mit einer aufgabengerechteren Ausstattung der Verkehrspolizisten. ${ }^{44}$ Übernahmen Polizeikräfte bereits seit der letzten Dekade des 19. Jahrhunderts zunehmend Wohlfahrtsaufgaben, so trat im Verkehrsbereich die volkspädagogische Stoßrichtung - in Form von Maßnahmen zur schulischen Verkehrserziehung - erst nach dem Ersten Weltkrieg stärker in den Vordergrund. ${ }^{45} \mathrm{Im}$,Zeitalter des Verkehrs" musste autogerechtes Verhalten schließlich von Motorisierten wie Unmotorisierten internalisiert werden, um die Straßen bei zunehmendem Motorisierungsgrad befahrbar zu halten.

Bei Eingriffen in den Autoverkehr war im Gegensatz zu anderen Interventionsbereichen im Kaiserreich keine einseitige Orientierung an den Bedürfnissen der gesellschaftlich führenden Schicht zu beobachten. Da es vorrangig die besitzenden Bürger waren, die dem Obrigkeitsstaat als Autofahrer begegneten, richtete sich der „repressive Zugriff“ hier gerade gegen eine „parteiliche, allgemeinverbindliche Festschreibung bürgerlicher Verhaltensmuster ${ }^{646}$, die sich in Geschwindigkeitsübertretungen und den verschiedensten Regelverletzungen manifestierten. Dabei stand in Deutschland noch in den Weimarer Jahren eine vergleichsweise geringe Zahl von spezialisierten Polizeikräften zur Verfügung. Während 1924 in London 7100 Beamte den Verkehr überwachten, waren es in Berlin nur 220, die sich in der Großstadt bei traditionell angespannter Sicherheitslage schwer taten, wilde Autofahrer, renitente Kutscher und undisziplinierte Fußgänger in Schach zu halten. ${ }^{47}$

Anschrift des Verfassers: Dr. Uwe Fraunholz, Technische Universität Dresden, Institut für Geschichte der Technik und der Technikwissenschaften, D-01062 Dresden, E-mail: Uwe.Fraunholz@mailbox.tu-dresden.de

44 Vor dem Ersten Weltkrieg blieben aber gerade die Beamten der Ortspolizeibehörden im Sinne eines umfassenden Polizei-Begriffs als Generalisten für „Sicherheit und Ordnung“ zuständig. Vgl. Herbert Reinke, ,... hat sich ein politischer und wirtschaftlicher Polizeistaat entwickelt". Polizei und Großstadt im Rheinland vom Vorabend des Ersten Weltkrieges bis zum Beginn der zwanziger Jahre, in: Alf Lüdtke (wie Anm. 27), S. 219-242, S. 220ff. Die ersten Polizeischulen wurden in Deutschland bereits um die Wende zum 20. Jahrhundert gegründet. Vgl. Robert Harnischmacher u. Arved Semerak, Deutsche Polizeigeschichte, Eine allgemeine Einführung in die Grundlagen, Stuttgart 1986, S. 70.

45 Zur schulischen Verkehrserziehung in den 1920er Jahren und dem Engagement der Polizei vgl. Dietmar Fack (wie Anm. 12), S. 285-300.

46 Vgl. Ralph Jessen, Polizei, Wohlfahrt und die Anfänge des modernen Sozialstaats in PreuBen während des Kaiserreichs, in: Geschichte und Gesellschaft 20, 1994, Nr. 2, S. 157-180.

47 Vgl. Bundesarchiv, R 1501, Nr. 14144, Niederschrift über die Sitzung des Beirats für das Kraftfahrwesen, 16. 12. 1924. 